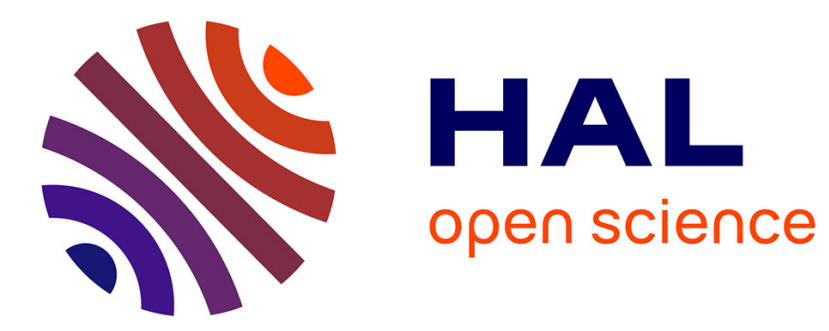

\title{
Poromechanics of microporous media
}

Laurent Brochard, Matthieu Vandamme, Roland J. M. Pellenq

\section{To cite this version:}

Laurent Brochard, Matthieu Vandamme, Roland J. M. Pellenq. Poromechanics of microporous media. Journal of the Mechanics and Physics of Solids, 2012, 60 (4), pp.606-622. hal-00691798

\section{HAL Id: hal-00691798 \\ https://hal.science/hal-00691798}

Submitted on 28 Jun 2018

HAL is a multi-disciplinary open access archive for the deposit and dissemination of scientific research documents, whether they are published or not. The documents may come from teaching and research institutions in France or abroad, or from public or private research centers.
L'archive ouverte pluridisciplinaire HAL, est destinée au dépôt et à la diffusion de documents scientifiques de niveau recherche, publiés ou non, émanant des établissements d'enseignement et de recherche français ou étrangers, des laboratoires publics ou privés. 


\title{
Poromechanics of Microporous Media
}

\author{
L. Brochard ${ }^{a}, \underline{\text { M. Vandamme }}{ }^{b}$, R.J.-M. Pellenq ${ }^{c, d}$
}

(a) Laboratoire Navier (École des Ponts ParisTech/IFSTTAR/CNRS), Université Paris-Est, École des Ponts ParisTech, 6-8 Av. B. Pascal, 77420 Champs-sur-Marne, France

(b) Laboratoire Navier (École des Ponts ParisTech/IFSTTAR/CNRS), Université Paris-Est, École des Ponts ParisTech, 6-8 Av. B. Pascal, 77420 Champs-sur-Marne, France Corresponding author Phone: +33 1641537 04; Fax: +33164153741

E-mail address: matthieu.vandamme@enpc.fr

(c) Centre Interdisciplinaire des Nanosciences de Marseille, Centre National de la Recherche Scientifique and Marseille Université, Campus de Luminy, Marseille, 13288 Cedex 09, France

(d) Department of Civil and Environmental Engineering, Massachusetts Institute of Technology, 77 Massachusetts Avenue, Cambridge, MA 02139

December 16, 2011 


\begin{abstract}
Microporous media, i.e., porous media made of pores with a nanometer size, are important for a variety of applications, for instance for sequestration of carbon dioxide in coal, or for storage of hydrogen in metal-organic frameworks. In a pore of nanometer size, fluid molecules are not in their bulk state anymore since they interact with the atoms of the solid: they are said to be in an adsorbed state. For such microporous media, conventional poromechanics breaks down.

In this work we derive poroelastic constitutive equations which are valid for a generic porous medium, i.e., even for a porous medium with pores of nanometer size. The complete determination of the poromechanical behavior of a microporous medium requires knowing how the amount of fluid adsorbed depends on both the fluid bulk pressure and the strain of the medium. The derived constitutive equations are validated with the help of molecular simulations on one-dimensional microporous media. Even when a microporous medium behaves linearly in the absence of any fluid (i.e., its bulk modulus does not depend on strain), we show that fluid adsorption can induce non linear behavior (i.e., its drained bulk modulus can then depend significantly on strain). We also show that adsorption can lead to an apparent Biot coefficient of the microporous medium greater than unity or smaller than zero.

The poromechanical response of a microporous medium to adsorption significantly depends on the pore size distribution. Indeed, the commensurability (i.e., the ratio of the size of the pores to that of the fluid molecules) proves to play a major role. For a onedimensional model of micropores with a variety of pore sizes, molecular simulations show that the amount of adsorbed fluid depends linearly on the strain of the medium. We derive linearized constitutive equations which are valid when such a linear dependence of the adsorbed amount of fluid on the strain is observed.

As an application, the case of methane and coal is considered. Molecular simulations of an adsorption of methane on a microporous realistic model for coal are performed with a flexible solid skeleton. The applicability of the set of linearized constitutive equations to this case is discussed and the results are shown to be consistent with swelling data measured during a classical adsorption experiment.
\end{abstract}

Keywords: Porous material (B), constitutive behavior (B), adsorption, molecular simulations

\title{
Introduction
}

Adsorption of fluid molecules in a microporous medium can cause the medium to swell or deform. Several applications require to better understand and model such a coupling between adsorption and deformation: underground coal swells when injected with carbon dioxide, thus inducing a loss of permeability of the coal bed reservoir and impairing the economic viability of such carbon dioxide geological sequestration schemes (Harpalani and Schraufnagel, 1990; Levine, 1996; Mazumder and Wolf, 2008; Ottiger et al., 2008, Vandamme et al., 2010); metal-organic frameworks (MOF) can be used for hydrogen storage (Murray et al., 2009) but can be subject to elastic deformation and to structural transitions upon adsorption of fluid (Coudert et al., 2008; Neimark et al., 2011); swellable organically modified silica (SOMS) can be used for water purification and swell when adsorbing the contaminant (Edmiston and Underwood, 2009); a kitchen sponge shrinks upon drying. This work focuses on the poromechanical behavior of such microporous media.

Not all porous media are microporous: pores in a porous medium can be classified into macropores, mesopores and micropores, depending on their size. Typically, the diameter of a macropore is greater than $50 \mathrm{~nm}$ (i.e., much greater than the characteristic range of intermolecular forces), that of a micropore is smaller than $2 \mathrm{~nm}$ (which is on the order of the characteristic range of intermolecular forces), and that of a mesopore is in-between. In a macropore, surface effects can be neglected, and all fluid molecules in the macropore are in their bulk state. For a poroelastic medium made of macropores, usual poromechanical 
constitutive equations link a confining stress $\sigma$, deviatoric stresses $s_{i j}$, and a fluid pore pressure $p$ to a variation $\varphi$ of porosity $\left(\varphi=\phi-\phi_{0}\right.$, where $\phi$ is the actual porosity and $\phi_{0}$ is the porosity in the state of reference), a volumetric strain $\epsilon$ and deviatoric strains $e_{i j}$ of the medium (Biot, 1941; Coussy, 2004):

$$
\begin{aligned}
\sigma & =K \epsilon-b p \\
\varphi & =\phi-\phi_{0}=b \epsilon+p / N \\
s_{i j} & =2 G e_{i j}
\end{aligned}
$$

where $K, G, b, N$ are the bulk modulus, shear modulus, Biot coefficient, and Biot modulus of the medium, respectively.

In a mesopore, not all fluid molecules are in their bulk state: a significant amount of fluid molecules is adsorbed at the surface of the mesopore. Therefore, in a mesoporous medium, surface effects must be taken into account. By introducing the interface energy $\gamma$ of the porefluid interface (i.e., of the surface of the pore), the specific area $s$ of the pores, the excess amount $\Gamma$ of fluid adsorbed per unit area of pore-fluid interface and the surface stress $\tilde{\sigma}^{s}$ which acts at the surface of the pore, the classical poroelastic constitutive equations can be extended to surface effects (for more details, see Vandamme et al. (2010) or Coussy (2010)):

$$
\begin{aligned}
\sigma & =K \epsilon-b p+\left.\tilde{\sigma}^{s} \frac{\partial s}{\partial \epsilon}\right|_{p} \\
\phi-\phi_{0} & =b \epsilon+\frac{p}{N}-\left.\tilde{\sigma}^{s} \frac{\partial s}{\partial p}\right|_{\epsilon} \\
s_{i j} & =2 G e_{i j}
\end{aligned}
$$

where the surface stress $\tilde{\sigma}^{s}$ is governed by Shuttleworth's equation (Shuttleworth, 1950) and by Gibbs' adsorption isotherm (Gibbs, 1928):

$$
\begin{aligned}
\tilde{\sigma}^{s} & =\gamma+\left.s \frac{\partial \gamma}{\partial s}\right|_{p}=\left.\frac{\partial(\gamma s)}{\partial s}\right|_{p} \\
\left.\frac{\partial \gamma}{\partial \mu}\right|_{s} & =-\Gamma
\end{aligned}
$$

In contrast, the size of a micropore is on the order of the range of molecular interactions, and therefore all fluid molecules in a micropore interact with the atoms of the solid matrix: fluid molecules are not in their bulk state anymore but are said to be adsorbed. For a microporous medium, all constitutive equations given above break down, since even the basic concepts of porous volume or specific surface of the pores become ambiguous: indeed, from one probe molecule to another, adsorption experiments provide different estimates for those parameters (Pellenq and Levitz, 2002; Bae and Bhatia, 2006).

The notion of disjoining pressure is a first step toward understanding the mechanical effect of adsorption in slit-like micropores. The disjoining pressure, also called solvation pressure, is the difference between the mechanical pressure acting on a thin film of fluid in a slit-like pore and the bulk pressure of the fluid. A disjoining pressure appears when the thickness of the film is on the order of the range of the molecular interactions. Reported values of disjoining pressures can be positive (disjoining) or negative (joining); their magnitude can be much larger than the bulk pressure of the fluid. For instance, in the case of water in clays, Malani et al. (2009) computed disjoining pressures up to a few GPa for a film of water confined between silica surfaces. Regarding the adsorption of methane in coal, Yang et al. 
(2010) computed disjoining pressures up to $200 \mathrm{MPa}$ at temperatures and pressures representative of underground conditions $(p \approx 10 \mathrm{MPa})$. Such high pressures can significantly affect the behavior of porous media. Models have been proposed to relate the disjoining pressures acting in such thin films to the overall mechanical behavior of a porous medium made of slit-like micropores subjected to adsorption. The model of Kowalczyk et al. (2008) predicts the overall behavior from the knowledge of the disjoining pressure, the pore size distribution, and the pore stiffness. Yang et al. (2010) applied this model to the case of coal swelling, assuming an incompressible solid phase, for definite pore sizes and showed that the swelling is highly sensitive to the size of the micropore. A different approach was proposed by Coudert et al. (2008), based on stability considerations in the osmotic statistical ensemble (isobaric-isothermal for the solid matrix and grand canonical for the adsorbing fluid). The thermodynamic framework they developed was used to determine the adsorption-induced opening of flexible materials. Mushrif and Rey (2009) proposed a model that tentatively combines adsorption and poromechanics but does not take into account the coupling between adsorption and deformation, even though deformation can have a significant effect on adsorption (Grosman and Ortega, 2008, 2009). In contrast, this coupling is well taken into account in the model developed by Neimark et al. (Ravikovitch and Neimark, 2006; Gor and Neimark, 2011; Neimark et al., 2011), which links the stress induced by adsorption to the grand thermodynamic potential of the adsorbed phase. In a recent work, Pijaudier-Cabot et al. (2011) revisited poromechanics in the context of microporous solids. They derive constitutive equations by introducing the density of the adsorbed phase, although such a notion is ambiguous in micropores with an ill-defined volume (Pellenq and Levitz, 2002; Bae and Bhatia, 2006). They also assume a simple relationship between the pressure induced by the adsorbed fluid and the isotherm of adsorption.

In this work, we aim at extending the realm of poromechanics to microporous media. In a first section, poroelastic constitutive equations are derived, which are valid for a generic porous medium. We check that the derived constitutive equations are consistent with constitutive equations already existing for macroporous and mesoporous media. In a second section, we perform molecular simulations of fluid adsorption in deformable one-dimensional microporous media. Molecular simulations allow validating the derived poroelastic constitutive equations. The effect of pore size distribution on the adsorption-induced strain is studied. In a last section, we perform molecular simulations of adsorption of methane in a deformable three-dimensional realistic coal structure. The applicability of the derived constitutive equations to adsorption in coal is discussed and the swelling predicted numerically is compared with experimental data.

\section{Thermodynamic approach of adsorption in a microp- orous medium}

\subsection{Derivation of constitutive equations}

We consider a porous medium with a generic pore size distribution. In this medium we consider a small representative volume, for which the Helmholtz free energy per unit undeformed volume is noted $f$. Energy can be provided to the system either by deforming it or by adding fluid molecules to it:

$$
d f=\sigma d \epsilon+s_{i j} d e_{i j}+\mu d n
$$

where $n$ is the Lagrangian molar concentration of fluid molecules per unit volume of porous medium (i.e., $n=N / V_{0}$ where $N$ is the actual number of moles of molecules in the medium and $V_{0}$ is the volume of the undeformed medium) and where $\mu$ is their molar chemical potential. The above equation can be rewritten as follows: 


$$
d(f-n \mu)=\sigma d \epsilon+s_{i j} d e_{i j}-n d \mu
$$

for which the use of Maxwell relations yields:

$$
\begin{gathered}
\left.\frac{\partial^{2}(f-n \mu)}{\partial \mu \partial \epsilon}\right|_{e_{i j}}=\left.\left.\frac{\partial^{2}(f-n \mu)}{\partial \epsilon \partial \mu}\right|_{e_{i j}} \Rightarrow \frac{\partial \sigma}{\partial \mu}\right|_{\epsilon, e_{i j}}=-\left.\frac{\partial n}{\partial \epsilon}\right|_{e_{i j}, \mu} \\
\left.\frac{\partial^{2}(f-n \mu)}{\partial \mu \partial e_{i j}}\right|_{\epsilon}=\left.\left.\frac{\partial^{2}(f-n \mu)}{\partial e_{i j} \partial \mu}\right|_{\epsilon} \Rightarrow \frac{\partial s_{i j}}{\partial \mu}\right|_{\epsilon, e_{i j}}=-\left.\frac{\partial n}{\partial e_{i j}}\right|_{\epsilon, \mu}
\end{gathered}
$$

Under the hypothesis of small deformations, if the porous material is isotropic, the adsorption of fluid molecules does not depend on the deviatoric strain: $\partial n /\left.\partial e_{i j}\right|_{\epsilon, \mu}=0$. If the material behaves linearly in vacuum (i.e., its bulk modulus $K$ and its shear modulus $G$ are constant), an integration of the above equations with respect to $\epsilon, e_{i j}$, and $\mu$ yields the following constitutive equations:

$$
\begin{aligned}
\sigma & =K \epsilon-\frac{\partial}{\partial \epsilon}\left[\int_{-\infty}^{\mu} n d \mu\right]_{\mu, e_{i j}=0} \\
n & =n(\epsilon, \mu) \\
s_{i j} & =2 G e_{i j}
\end{aligned}
$$

In the above equations, the amount of fluid in the medium appears naturally as a state variable of the system (Rice and Cleary, 1976; Coussy, 1995). Eq. (13) is consistent with that obtained by Gor and Neimark (2011).

Using the Gibbs-Duhem relation in isothermal conditions $\left(\mathrm{d} \mu=\bar{V}_{b} \mathrm{~d} p\right.$, where $\bar{V}_{b}$ is the molar volume of the bulk fluid), the constitutive equations can be rewritten in an alternative form by introducing the bulk pressure $p$ of the fluid as a state variable:

$$
\begin{aligned}
\sigma & =K \epsilon-\frac{\partial}{\partial \epsilon}\left[\int_{0}^{p} n \bar{V}_{b} d p\right]_{p, e_{i j}=0} \\
n & =n(\epsilon, p) \\
s_{i j} & =2 G e_{i j}
\end{aligned}
$$

In the case of a fluid mixture, the energy balance (10) becomes:

$$
d\left(f-\sum_{i} n_{i} \mu_{i}\right)=\sigma d \epsilon+s_{i j} d e_{i j}-\sum_{i} n_{i} d \mu_{i}
$$

where $n_{i}$ and $\mu_{i}$ are the Lagrangian molar concentration and the molar chemical potential of the $i$-th component of the mixture. The associated Maxwell relations for each component are:

$$
\left.\frac{\partial \sigma}{\partial \mu_{i}}\right|_{\epsilon, e_{i j}, \mu_{j \neq i}}=-\left.\frac{\partial n_{i}}{\partial \epsilon}\right|_{e_{i j}, \mu_{1}, \ldots, \mu_{n}} \text { and }\left.\frac{\partial s_{i j}}{\partial \mu_{i}}\right|_{\epsilon, e_{i j}, \mu_{j \neq i}}=-\left.\frac{\partial n_{i}}{\partial e_{i j}}\right|_{\epsilon, \mu_{1}, \ldots, \mu_{n}}
$$

The constitutive equations in the case of fluid mixtures are obtained by successive integration of the Maxwell relations with respect to the chemical potentials $\mu_{i}$ :

$$
\begin{aligned}
\sigma & =K \epsilon-\frac{\partial}{\partial \epsilon}\left[\int_{(-\infty, \ldots,-\infty)}^{\left(\mu_{1}, \ldots, \mu_{n}\right)}\left(\sum_{i} n_{i}\right) d \mu_{1} \ldots d \mu_{n}\right]_{\mu_{1}, \ldots, \mu_{n}, e_{i j}=0} \\
n_{i} & =n_{i}\left(\epsilon, \mu_{1}, \ldots, \mu_{n}\right) \\
s_{i j} & =2 G e_{i j}
\end{aligned}
$$


where the integration does not depend on the chosen path.

\subsection{Consistency with usual poromechanics}

We aim at verifying whether the general constitutive equation (16) derived in the previous section for a generic porous medium is consistent with constitutive equations already existing for macroporous media (Eq. (1)) and for mesoporous media (Eq. (7)).

In a macroporous medium, a porosity $\phi$ can be well identified. When the material is in contact with a reservoir of fluid at pressure $p$, the porosity is filled with fluid molecules in their bulk state, from what follows $\phi=n \bar{V}_{b}$, so that the constitutive equation (16) can be rewritten as:

$$
\sigma=K \epsilon-\frac{\partial}{\partial \epsilon}\left[\int_{0}^{p} \phi d p\right]_{p}
$$

where the fact that the deviatoric strains must be kept constant during the differentiation is omitted from the notation.

Inserting the macroporous constitutive equation (2) into the equation above, we retrieve the macroporous constitutive equation (1). This verification shows that the general constitutive equations derived in this work are consistent with usual poromechanics, which is valid for macroporous media only.

In contrast, in a mesoporous medium, fluid molecules (their molar concentration per unit volume of undeformed porous medium is noted $n$ ) are either in bulk form (their molar concentration per unit volume of undeformed porous medium is noted $n_{b}$ ) in the mesopores, or adsorbed (their molar concentration per unit volume of undeformed porous medium is noted $n_{a}$ ) at the surface of the mesopores, from what follows: $n=n_{a}+n_{b}$ and $n \bar{V}_{b}=\phi+n_{a} \bar{V}_{b}$. Therefore, for a mesoporous medium that behaves linearly, the general constitutive equation (16) can be rewritten as:

$$
\sigma=K \epsilon-\frac{\partial}{\partial \epsilon}\left[\int_{0}^{p} \phi d p\right]_{p}-\frac{\partial}{\partial \epsilon}\left[\int_{0}^{p} n_{a} \bar{V}_{b} d p\right]_{p}
$$

If we input the mesoporous constitutive equation (5) into the general constitutive equation (25) and note that $n_{a}=\Gamma s$, we obtain:

$$
\sigma=K \epsilon-b p+\frac{\partial}{\partial \epsilon}\left[\int_{0}^{p}\left(\left.\tilde{\sigma}^{s} \frac{\partial s}{\partial p}\right|_{\epsilon}-s \Gamma \bar{V}_{b}\right) d p\right]_{p}
$$

Gibbs' equation (7) can be rewritten:

$$
\left.\frac{\partial \gamma}{\partial p}\right|_{s}=-\Gamma \bar{V}_{b}
$$

and the integrand in Eq. (26) can be simplified by using the above expression and Shuttleworth's equation (8):

$$
\begin{aligned}
\left.\tilde{\sigma}^{s} \frac{\partial s}{\partial p}\right|_{\epsilon}-s \Gamma \bar{V}_{b} & =\left.\left(\gamma+\left.s \frac{\partial \gamma}{\partial s}\right|_{p}\right) \frac{\partial s}{\partial p}\right|_{\epsilon}+\left.s \frac{\partial \gamma}{\partial p}\right|_{s} \\
& =\left.\left.\frac{\partial(\gamma s)}{\partial s}\right|_{p} \frac{\partial s}{\partial p}\right|_{\epsilon}+\left.\frac{\partial(\gamma s)}{\partial p}\right|_{s}=\left.\frac{\partial(\gamma s)}{\partial p}\right|_{\epsilon}
\end{aligned}
$$

so that Eq. (26) can be rewritten as:

$$
\sigma=K \epsilon-b p+\left.\frac{\partial(\gamma s)}{\partial \epsilon}\right|_{p}=K \epsilon-b p+\left.\tilde{\sigma}^{s} \frac{\partial s}{\partial \epsilon}\right|_{p}
$$


which is the mesoporous constitutive equation (4). This verification shows that the general constitutive equations here derived are consistent with regular poromechanics extended to surface effects, which is valid for mesoporous materials only (Vandamme et al., 2010).

\subsection{Poroelastic properties of the porous medium}

The general poroelastic constitutive equations (13)-(15), or equivalently (16)-(18), are valid for porous media with a generic pore size distribution. In particular, they still hold when the porosity is made of micropores, for which even the notion of pore volume becomes ambiguous.

The general constitutive equations here derived can be used to calculate apparent poroelastic properties. For instance, one can define an apparent tangent drained bulk modulus $K^{t}=\partial \sigma /\left.\partial \epsilon\right|_{p}$, which, with the help of the constitutive equation (16), is given by:

$$
K^{t}(\epsilon, p)=\left.\frac{\partial \sigma}{\partial \epsilon}\right|_{p}=K-\frac{\partial^{2}}{\partial \epsilon^{2}}\left[\int_{0}^{p} n \bar{V}_{b} d p\right]_{p}=K-\frac{\partial^{2}}{\partial \epsilon^{2}}\left[\int_{-\infty}^{\mu} n d \mu\right]_{\mu}
$$

Such an equation interestingly shows that, if the adsorption isotherm $n(\epsilon, p)$ (or, equivalently, $n(\epsilon, \mu)$ ) depends on the deformation in a complex manner, the apparent drained tangent bulk modulus $K^{t}$ of the porous medium may differ from its bulk modulus $K$ in vacuum: the coupling between adsorption and deformation can lead to apparent non-linearities of the porous medium, even when the porous medium behaves linearly in the absence of any fluid.

Likewise, one can define an apparent tangent Biot coefficient $b^{t}=-\partial \sigma /\left.\partial p\right|_{\epsilon}$, which, with the help of the constitutive equation (16), is given by:

$$
b^{t}(\epsilon, p)=-\left.\frac{\partial \sigma}{\partial p}\right|_{\epsilon}=\left.\frac{\partial\left(n \bar{V}_{b}\right)}{\partial \epsilon}\right|_{p}=\left.\frac{\partial\left(n \bar{V}_{b}\right)}{\partial \epsilon}\right|_{\mu}
$$

For a linear poroelastic material made of macropores, a relationship exists between bulk modulus and Biot coefficient (Coussy, 2004): $b=1-K / K_{s}$, where $K_{s}$ is the bulk modulus of the solid phase. For a microporous medium, such a relation no more holds and it is even not possible to define a solid bulk modulus $K_{s}$ unambiguously. Nevertheless, combining Eqs. (31) and (32), one can readily derive the following relation:

$$
K^{t}(\epsilon, p)=K-\frac{\partial}{\partial \epsilon}\left[\int_{0}^{p} b^{t}(\epsilon, p) d p\right]_{p}
$$

which shows that the tangent drained bulk modulus $K^{t}$ and the tangent Biot coefficient $b^{t}$ are linked with each other.

Equivalently, one can define apparent secant poroelastic properties. For instance, one can introduce an apparent secant drained bulk modulus $K^{s}=[\sigma(\epsilon, p)-\sigma(\epsilon=0, p)] / \epsilon$, which, with the help of the constitutive equation (16), is given by:

$$
K^{s}(\epsilon, p)=\frac{\sigma(\epsilon, p)-\sigma(\epsilon=0, p)}{\epsilon}=K-\frac{1}{\epsilon} \int_{0}^{\epsilon} \frac{\partial^{2}}{\partial \epsilon^{2}}\left[\int_{0}^{p} n \bar{V}_{b} d p\right]_{p} d \epsilon
$$

As was the case for the apparent tangent properties, if the adsorption isotherm $n(\epsilon, p)$ (or, equivalently, $n(\epsilon, \mu)$ ) depends on the deformation in a complex manner, the apparent drained secant bulk modulus $K^{s}$ of the porous medium may differ from its bulk modulus $K$ in vacuum.

One can also define an apparent secant Biot coefficient $b^{s}=-[\sigma(\epsilon, p)-\sigma(\epsilon, p=0)] / p$, which, with the help of the constitutive equation (16), is given by:

$$
b^{s}(\epsilon, p)=-\frac{\sigma(\epsilon, p)-\sigma(\epsilon, p=0)}{p}=\frac{1}{p}\left[\frac{\partial}{\partial \epsilon} \int_{0}^{p} n \bar{V}_{b} d p\right]_{p}
$$


From a combination of the two equations above, one can readily derive the following relation:

$$
K^{s}(\epsilon, p)=K-\frac{p}{\epsilon}\left[b^{s}(\epsilon, p)-b^{s}(\epsilon=0, p)\right]
$$

which shows that the secant drained bulk modulus $K^{s}$ and the secant Biot coefficient $b^{s}$ are linked with each other.

\subsection{Linearized poroelastic constitutive equations}

Under the hypothesis of small deformations $(\epsilon \ll 1)$, we can perform a first-order expansion of the adsorption isotherm:

$$
n(\epsilon, p) \approx n^{0}(p)(1+C(p) \epsilon)
$$

where $n^{0}(p)=n(\epsilon=0, p)$ is the adsorption isotherm when the medium is kept at zero deformation. If the above approximation holds, the constitutive equation (16) can be rewritten as:

$$
\sigma=K \epsilon-f(p)=K \epsilon-\int_{0}^{p} C(p) n^{0}(p) \bar{V}_{b}(p) d p
$$

For specific porous media, it may well be that the coupling coefficient $C(p)$ does not depend on the pressure of the fluid (i.e., $C(p)=C$ ). In such a case, the poroelastic constitutive equation (38) further simplifies:

$$
\sigma=K \epsilon-C \int_{0}^{p} n^{0}(p) \bar{V}_{b}(p) d p
$$

For instance, the above equation would hold for a macroporous medium made of an incompressible solid skeleton. Indeed, for such a medium, we infer from the constitutive equation (2) that the amount $n(\epsilon, p)$ of fluid in the porous medium can be expressed as: $n(\epsilon, p)=n(\epsilon=0, p)+\rho \epsilon=n^{0}(p)\left(1+\epsilon \rho / n^{0}(p)\right)$, where $\rho$ is the molar density of the fluid. Therefore, for a macroporous medium made of an incompressible solid skeleton, the first-order expansion (37) of the adsorption isotherm is valid for any strain, with: $C=1 / \phi(\epsilon=0)$.

Practically, $K$ can be determined by performing a compression test on the sample. But the complete determination of the adsorption isotherm $n(\epsilon, p)$ would require to perform adsorption experiments at several fixed strain levels, which is very unpractical. Often, only one adsorption experiment is performed, in so-called unjacketed conditions: the porous medium is immersed in the bulk fluid and the pressure $p$ of the fluid is increased. In such an experiment, the molar concentration $n^{u}(p)$ of fluid molecules per unit volume of porous medium is measured; sometimes, over the same experiment, the volumetric strain $\epsilon^{u}(p)$ also is measured. In unjacketed conditions, the confining stress is equal to the opposite of the fluid pressure $(\sigma=-p)$ and the constitutive equation (16) yields a relation that the volumetric strain $\epsilon^{u}(p)$ must verify:

$$
-p=K \epsilon^{u}(p)-\frac{\partial}{\partial \epsilon}\left[\int_{0}^{p} n(\epsilon, p) \bar{V}_{b} \mathrm{~d} p\right]_{p, \epsilon=\epsilon^{u}}
$$

Assuming that the first order expansion of Eq. (37) is valid, a differentiation of Eq. (40) with respect to the pressure $p$ of the fluid yields:

$$
C(p)=\frac{1+K \mathrm{~d} \epsilon^{u} / \mathrm{d} p}{n^{u} \bar{V}_{b}-\epsilon^{u}\left(1+K \mathrm{~d} \epsilon^{u} / \mathrm{d} p\right)}
$$

The above equation shows how the coupling coefficient $C(p)$ needed in a first-order expansion of the adsorption isotherm can be fully determined from a regular adsorption experiment 
performed in unjacketed conditions in which both the adsorption isotherm $n^{u}(p)$ and the volumetric strain $\epsilon^{u}(p)$ would be measured. This equation also enables to check whether the coupling coefficient $C(p)$ depends or not on the bulk pressure of the fluid.

One should realize, however, that, if the first-order expansion (37) is not valid for the porous medium and for the fluid considered (i.e., in the general case), a regular adsorption experiment will not provide enough information in order to fully characterize the poromechanical behavior of the medium. Indeed, if Eq. (37) is not valid, the constitutive equation that governs the volumetric poromechanical behavior of the medium is Eq. (16) and not Eq. (39). This former equation can only be used if the adsorption isotherms $n(\epsilon, p)$ are known, which requires making the strain of the medium and the bulk pressure of the adsorbed fluid vary independently. Making those two parameters vary independently from each other can not be done in a regular adsorption experiment in which the sample is immersed in a fluid since, in such a case, the confining stress is equal to the opposite of the fluid pressure $(\sigma=-p)$.

\section{Case study: adsorption in one-dimensional microp- orous media}

In order to illustrate the effect of adsorption on the poromechanical behavior of a microporous medium and validate the derivations performed in the Sec. 1.1, we model one-dimensional microporous chain-like pores in which we perform Monte Carlo simulations of adsorption of fluid. All micropores of the first chain model studied have the same size (Sec. 2.1). We then study a chain made of micropores with a variety of sizes (Sec. 2.2). We will refer to the first chain as being 'crystalline' and to the second one as being 'amorphous'.

\subsection{Crystalline chain}

We considered a one dimensional chain made of equally distributed atoms separated by an equilibrium distance $r_{0}$. Each atom was interacting with its two closest neighbors. The energy $E_{i j}$ of interaction was harmonic $\left(E_{i j}=k\left(r_{i j}-r_{0}\right)^{2}\right.$ where $r_{i j}$ is the distance between the atoms $i$ and $j$ ), thus making this one-dimensional chain linear elastic. The space between two atoms of the solid was large enough to accommodate a few adsorbed fluid molecules (see Fig. 1). The fluid considered for the simulation was a Lennard-Jones fluid. The energy

of interaction between two fluid molecules was: $E_{i j}=4 \varepsilon_{F F}\left(\left(\sigma_{F F} / r_{i j}\right)^{12}-\left(\sigma_{F F} / r_{i j}\right)^{6}\right)$, where $\varepsilon_{F F}$ and $\sigma_{F F}$ are the Lennard-Jones parameters of the fluid. The energy of interaction between a fluid molecule and an atom of the chain was a Lennard-Jones potential as well, but with different parameters $\varepsilon_{F S}$ and $\sigma_{F S}$. We performed Monte Carlo molecular simulations of such a system (Allen and Tildesley, 1989; Frenkel and Smit, 2001). The solid was simulated in the Canonical ensemble (the number $N_{s}$ of atoms in the solid, the volume $V$ and the temperature $T$ were fixed). The fluid was simulated in the Grand canonical ensemble (the chemical potential $\mu$ of the fluid was fixed, the volume $V$ and the temperature $T$ were the same as for the solid). Since the simulations were one-dimensional, the volume $V$ was the length of the chain. During the simulations, we computed the confining stress with the Virial estimate (Allen and Tildesley, 1989) and the number $N_{f}$ of fluid molecules in the system. Several values of the fluid chemical potential $\mu$ and of the volume $V$ were considered. One Monte Carlo simulation was performed for each pair $(\mu, V)$.

The values of the different parameters used for this numerical experiment were chosen such that the adsorption was significant: $k=2 \times 10^{-20} \mathrm{~J} . \AA^{-2}, r_{0}=6 \AA, \varepsilon_{F F}=2 \times 10^{-21} \mathrm{~J}$, $\sigma_{F F}=1 \AA, \varepsilon_{F S}=5 \times 10^{-21} \mathrm{~J}, \sigma_{F S}=2 \AA$. The simulated chain was made of 50 atoms and was $300 \AA$ long at rest. The temperature $T$ of the system was fixed at $100 \mathrm{~K}$. We 


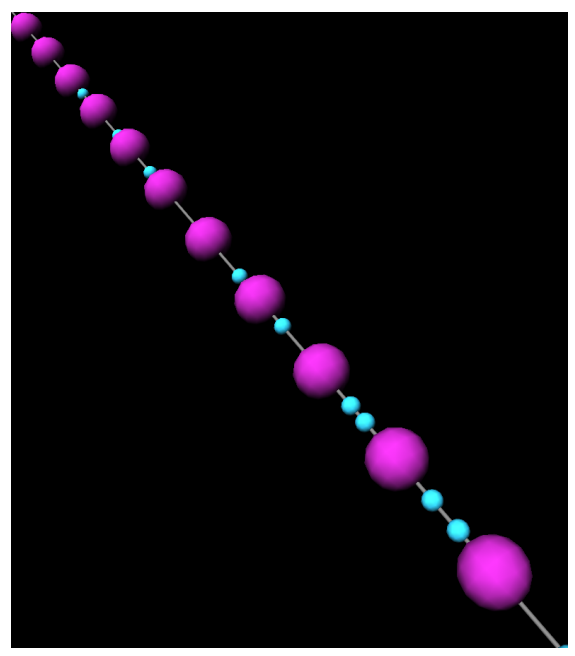

Figure 1: Example of molecular configuration. The fluid molecules can enter or leave the interstitial space between solid atoms. The atoms of the solid are the large pink balls, the fluid molecules are the small blue balls, the line represents the 1D space.

considered periodic boundary conditions and the atoms were interacting according to the minimum image convention.

In the following sections, we will refer to the dimensionless fluid pressure $p^{*}=p / K$ and to the dimensionless confining stress $\sigma^{*}=\sigma / K$, where $K=2 k r_{0}=2.4 \times 10^{-9} \mathrm{~N}$ is the bulk modulus of the chain at zero fluid pressure. Molecular simulations of the bulk fluid were performed to find the equation of state $p^{*}(\mu)$ of the fluid, which links the molar chemical potential $\mu$ of the fluid with its dimensionless bulk pressure $p^{*}$.

Figure 2 displays the amount $n(\epsilon, p)$ of fluid molecules per unit length of the undeformed chain in function of the strain $\epsilon$ of the chain for various dimensionless bulk fluid pressures $p^{*}$. At large pressures, the greater the strain was, the more fluid molecules there were in the pores. At low pressures, interestingly, the amount of fluid molecules in the pores decreased with the strain of the medium.

At zero pressure of the fluid $(\mu \rightarrow-\infty)$, there was no fluid molecule in the system and the elastic behavior of the medium was linear: $\sigma=\sigma_{0}(\epsilon)=K \epsilon$. But the apparent mechanical properties of the porous medium varied upon adsorption. For instance, we display in Fig. 3 the apparent tangent drained bulk modulus $K^{t}$ defined by Eq. (31). As is expected for an elastic medium, $K^{t}=K$ in the absence of any fluid. By contrast, under adsorption $K^{t}$ was no more constant: the adsorption of fluid modified its value by up to $-24 \%$ and $+45 \%$ in this example.

The fluid-induced stress $\sigma(\epsilon, p)-\sigma_{0}(\epsilon)=\sigma(\epsilon, p)-K \epsilon$ characterizes the confining stress that must be added to the system to keep it at a given strain $\epsilon$ and counteract an increase of fluid pressure $p$. We display in Fig. 4 the dimensionless fluid-induced stress $\sigma^{*}(\epsilon, p)-\sigma_{0}^{*}(\epsilon)=$ $\left(\sigma(\epsilon, p)-\sigma_{0}(\epsilon)\right) / K$ in function of the bulk fluid pressure for different strains: we display both the stress computed directly from the molecular simulations by using the Virial estimate and the stress calculated with the constitutive equation (16) and the simulated adsorption isotherm $n(\epsilon, p)$ displayed in Fig. 2. The match between those two ways of estimating the fluid-induced stress was very satisfactory, proving therefore that the derivations performed in Sec. 1.1 enable to capture accurately the poromechanical behavior of the chain here considered, although the adsorption isotherms strongly depend on the strain of the medium (see Fig. 2). 


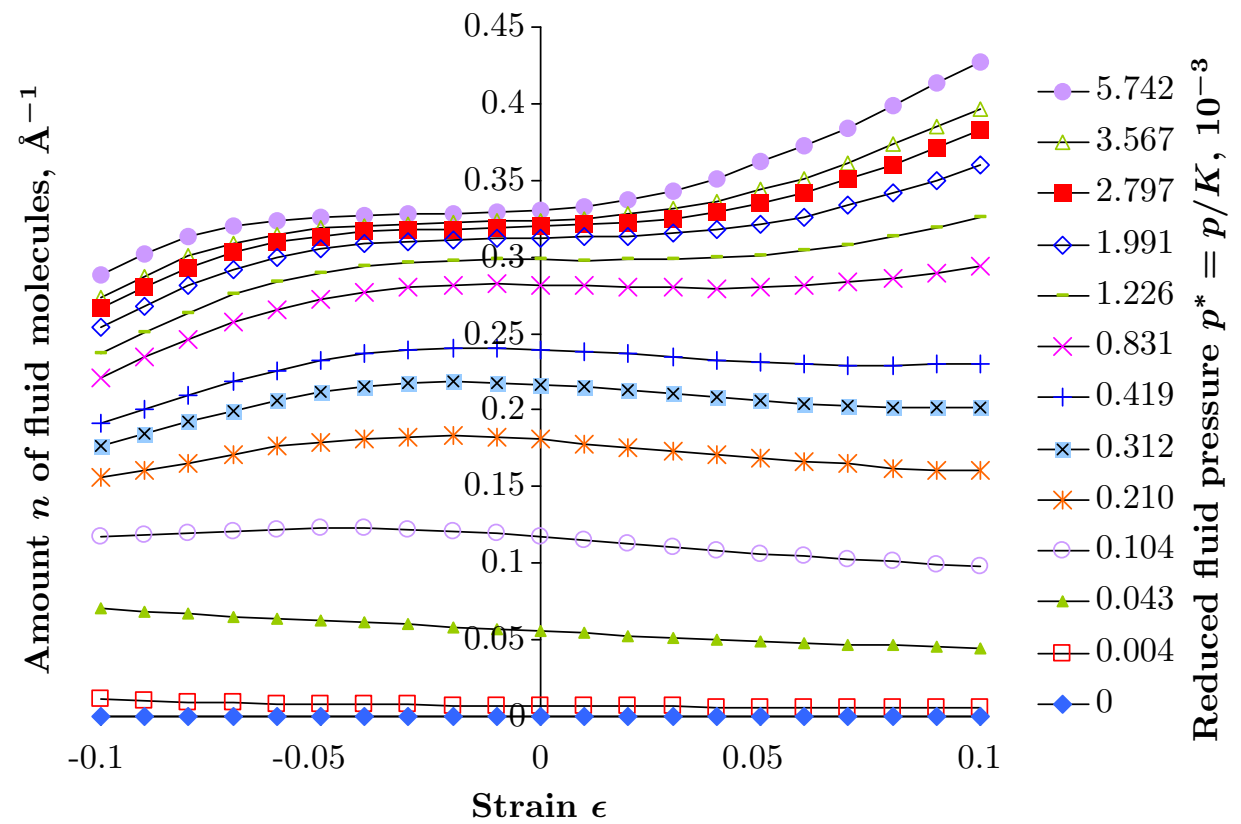

Figure 2: Amount of fluid molecules adsorbed per unit length of the undeformed chain, in function of the strain of the solid for various bulk pressures of the fluid.

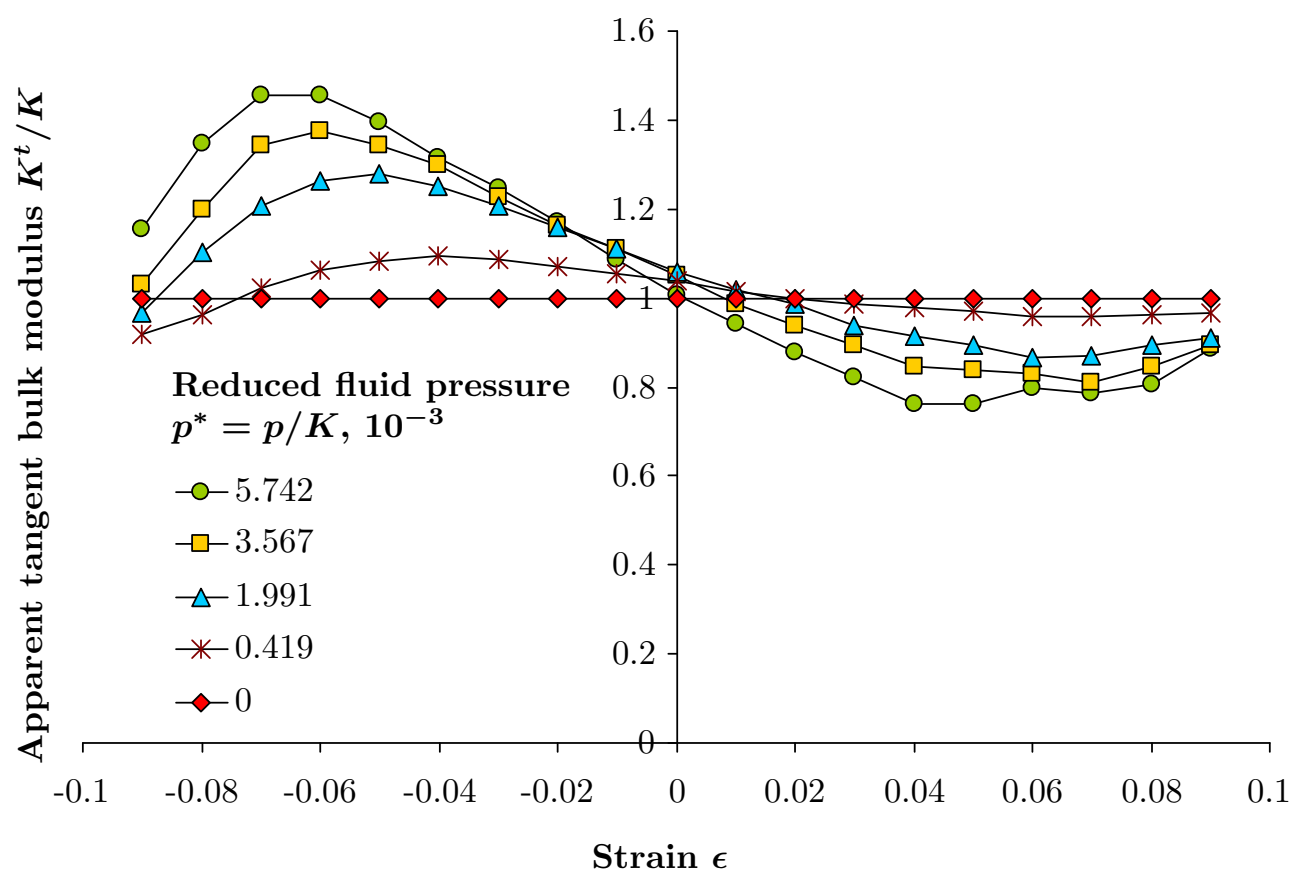

Figure 3: Apparent tangent drained bulk modulus $K^{t}$ of the crystalline chain. 


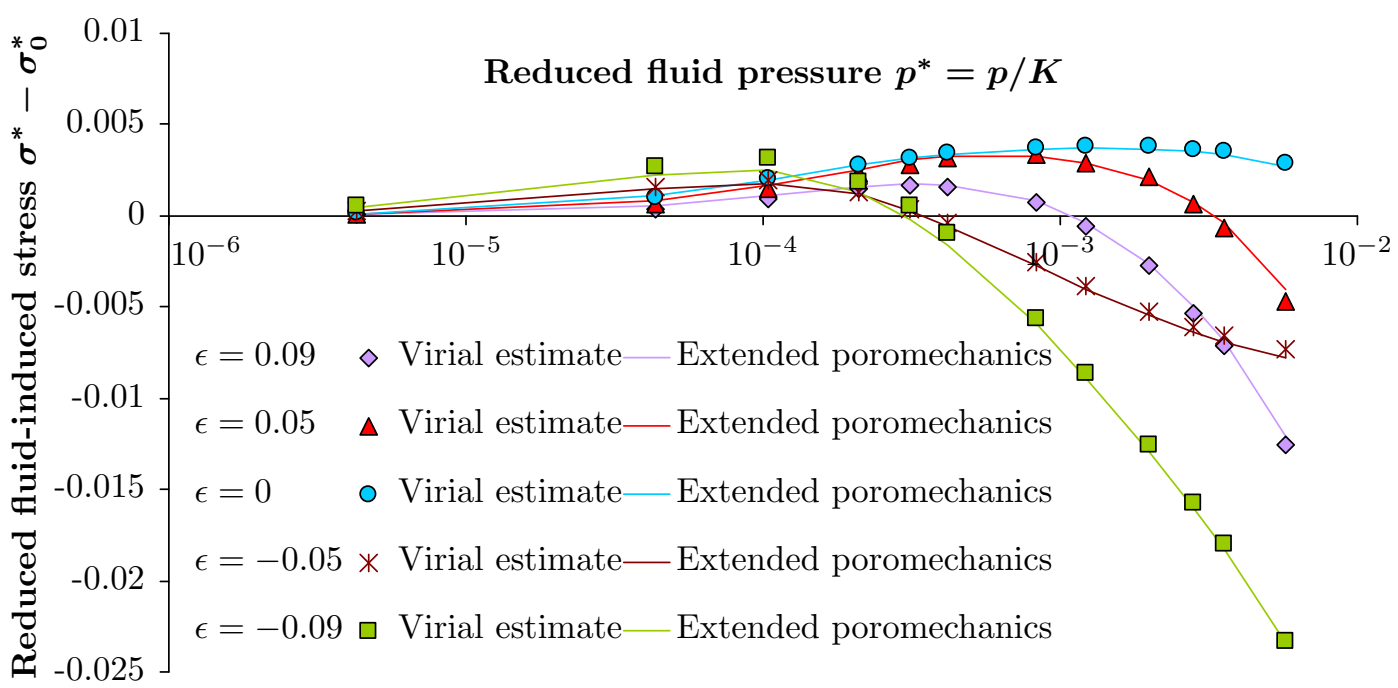

Figure 4: Dimensionless fluid-induced confining stress computed from the molecular simulations with the Virial estimate (symbols) and computed with the simulated adsorption isotherms $n(\epsilon, p)$ combined with the constitutive equation (13) (lines).

As can be observed in Fig. 4, the fluid-induced stress could be positive or negative and was much greater than the bulk pressure of the fluid. This is surprising since, for a macroporous medium, the poroelastic constitutive equation (1) shows that the fluid-induced stress is $\sigma(\epsilon, p)-\sigma_{0}(\epsilon)=-b p$, where $b$ is the Biot coefficient $(0<b \leq 1, b=1$ corresponding to an incompressible solid matrix). The apparent tangent and secant Biot coefficients are displayed in Fig. 5 and in Fig. 6, respectively. To a large extent, the values obtained for those Biot coefficients are out of the conventional range $[0 ; 1]$, reaching values as high as 10 and as low as -10 .

In the next section, adsorption on a one-dimensional microporous medium with a different pore size distribution is considered.

\subsection{Amorphous chain}

In this section we consider a one-dimensional microporous medium with a variety of micropore sizes chosen randomly. We will refer to this medium as to the 'amorphous' chain. The molecular interactions were the same as in Sec. 2.1, except for the equilibrium length $r_{0}$ which was specific to each pair of neighboring atoms of the solid. The equilibrium lengths were generated randomly between $3 \AA$ and $11 \AA$ following a uniform law (see Fig. 7). The chain was made of 139 atoms and its total length at rest was $1000 \AA$. We performed Monte Carlo simulations of adsorption of fluid on this chain, by using the same potentials of interaction as in Sec. 2.1.

For various bulk pressures of the fluid and strains of the medium, we computed the number of adsorbed molecules and the confining stress by using the Virial estimate. We display in Fig. 8 and in Fig. 9 the adsorbed amount $n(\epsilon, p)$ per unit volume of the undeformed chain, and the fluid-induced stress $\sigma(\epsilon, p)-\sigma(\epsilon, p=0)$ due to the adsorption of fluid, respectively. The behavior of the amorphous chain differed significantly from that of the crystalline chain (see Fig. 2 and Fig. 4). Indeed, for the amorphous chain, the adsorbed amounts of fluid depended linearly on the strain of the medium and the fluid-induced stress did almost not depend on the strain of the medium. Moreover, in contrast to what was observed for the crystalline chain (see Fig. 3) the apparent tangent bulk modulus $K^{t}$ remained mostly constant $\left(K^{t}=\right.$ 


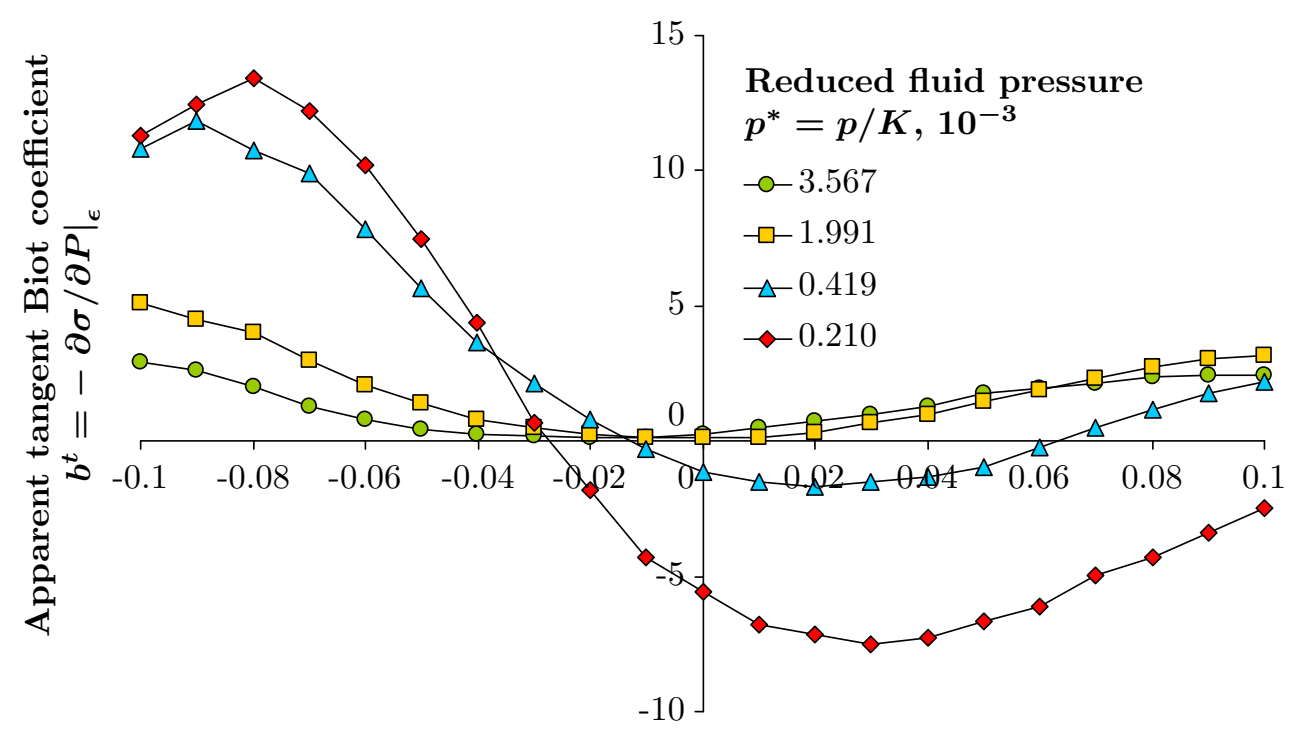

Strain $\epsilon$

Figure 5: Apparent tangent Biot coefficient $b^{t}$ of the crystalline chain.

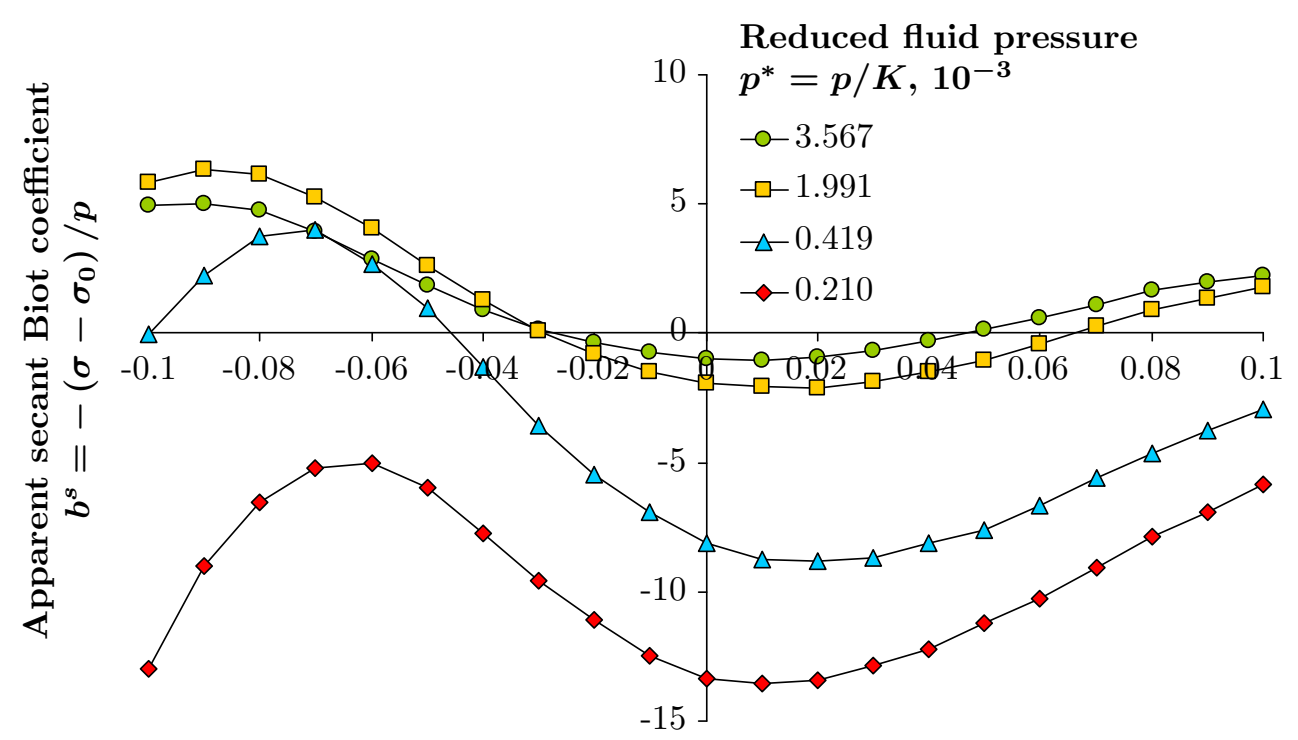

Strain $\epsilon$

Figure 6: Apparent secant Biot coefficient $b^{s}$ of the crystalline chain. 


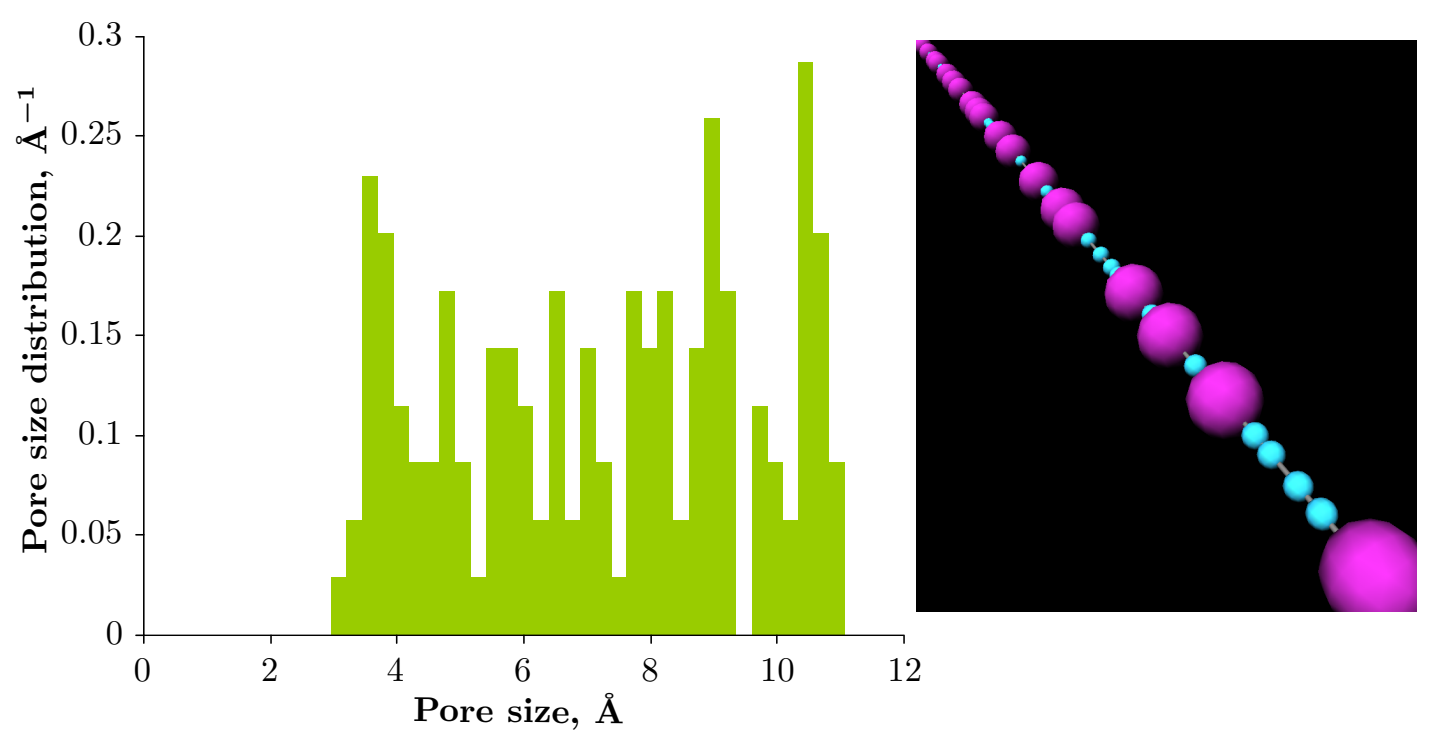

Figure 7: (Left) Pore size distribution for the amorphous chain; (Right) Example of molecular configuration.

$K \pm 1.8 \%$ ) for strains $\epsilon$ ranging from -0.1 to +0.1 and for dimensionless fluid pressures $p^{*}=p / K$ ranging from 0 to $5.7 \times 10^{-3}$.

We display in Fig. 9 both the fluid-induced stress computed directly from the molecular simulation by using the Virial estimate and the fluid-induced stress calculated with the constitutive equation (16) and the simulated adsorption isotherm $n(\epsilon, p)$ displayed in Fig. 8. As was the case for the crystalline chain (see Fig. 4), both ways of estimating the fluid-induced stress compared very well with each other, which further confirms that the constitutive equations derived in Sec. 1.1 enable to capture accurately the poromechanical behavior of a microporous medium.

We display in Fig. 10 the apparent secant Biot coefficient $b^{s}$. At low bulk fluid pressures $\left(p^{*}<10^{-3}\right)$, this coefficient significantly depended on the strain. The greater the bulk pressure of the fluid was, the less this coefficient depended on the strain, and the smaller its average value over strains from -0.1 to +0.1 was. At the largest bulk fluid pressure considered $\left(p^{*}=2.97 \times 10^{-3}\right)$, the apparent secant Biot coefficient was mostly insensitive to the strain $\left(b^{s}=1.91 \pm 15 \%\right)$. In any case, for all pressures considered, the secant Biot coefficient remained greater than the value observed in macroporous media $(b<1)$.

\subsection{Discussion}

The poromechanical behaviors of the crystalline chain (Sec. 2.1) and of the amorphous chain (Sec. 2.2) differed from each other and from that of a usual macroporous medium.

For the crystalline chain, the fluid-induced stress $\sigma(\epsilon, p)-\sigma_{0}(\epsilon)$ sometimes was positive or 'joining' (in the sense that adsorption made the medium shrink and should be counterbalanced by some additional tensile confining stress if one wanted to keep the strain constant) and sometimes was negative or 'disjoining' (in the sense that it made the medium swell). In fact, the fluid had a joining effect at low bulk pressure of the fluid, and mostly a disjoining effect at the highest bulk pressures considered (see Fig. 4). The complex effect of adsorption in this crystalline chain is closely related to the number of fluid molecules that micropores can accommodate. Indeed, by deforming the medium from $\epsilon=-0.1$ to $\epsilon=0.1$, the average distance between neighboring atoms of the solid changed by $1.2 \AA$, which is approximately the size of one fluid molecule. We display in Fig. 11 the fluid-induced stress $\sigma(\epsilon, p)-\sigma_{0}(\epsilon)$ 


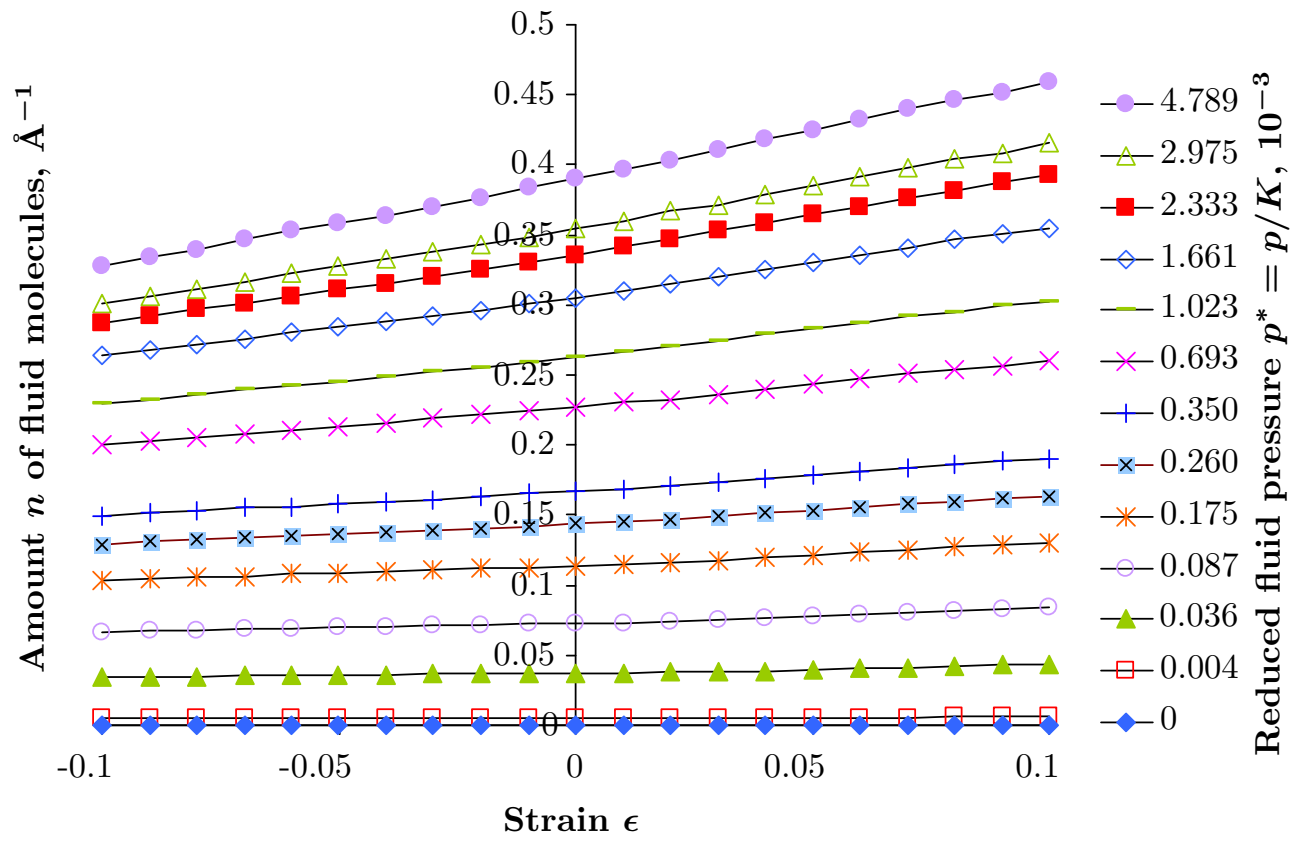

Figure 8: Adsorbed amount of fluid molecules in the amorphous chain per unit length of the undeformed chain.

\section{Reduced fluid pressure $p^{*}=p / K$}

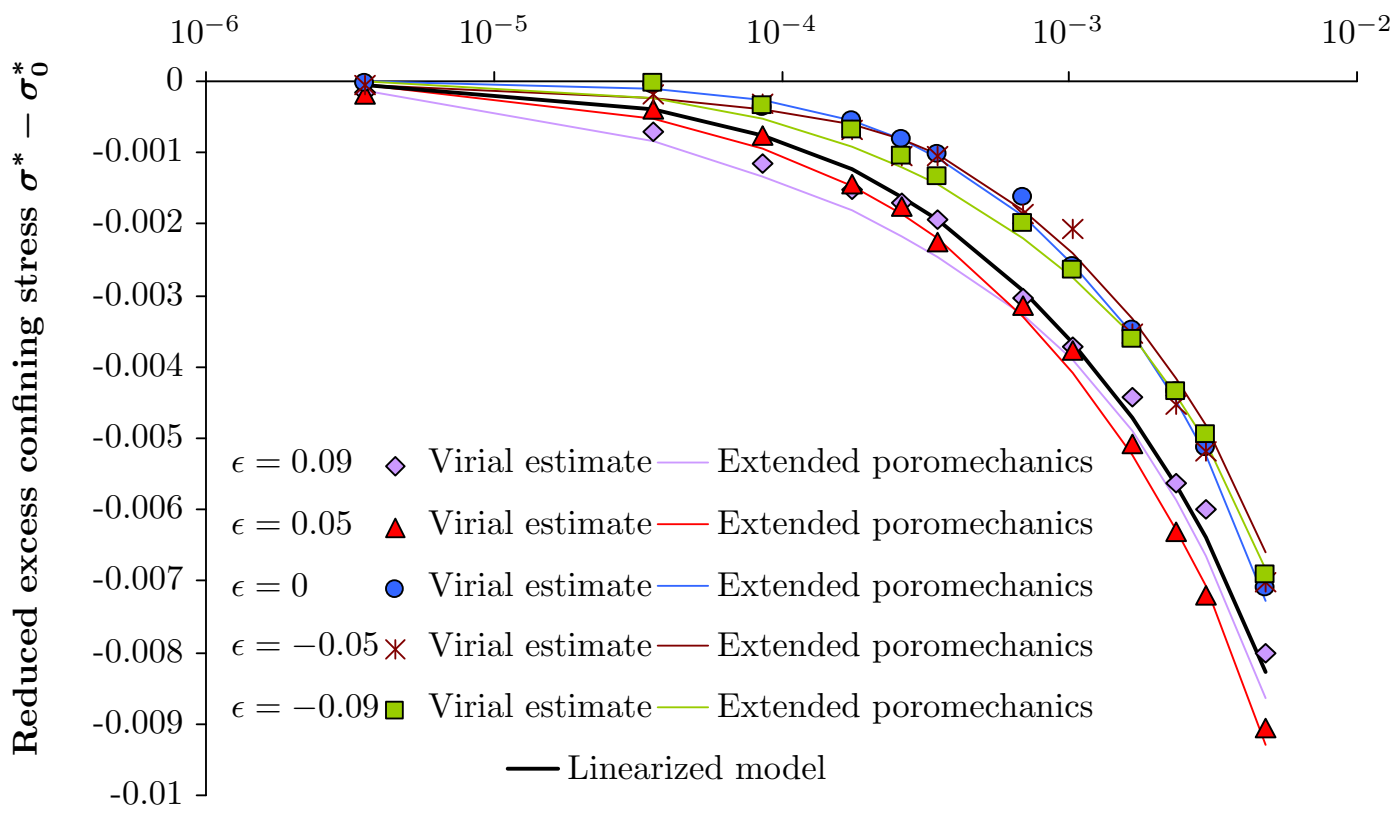

Figure 9: Dimensionless fluid-induced stress computed from the molecular simulations with the Virial estimate (symbols) and computed with the simulated adsorption isotherms $n(\epsilon, p)$ combined with the state equation (13) (lines). 


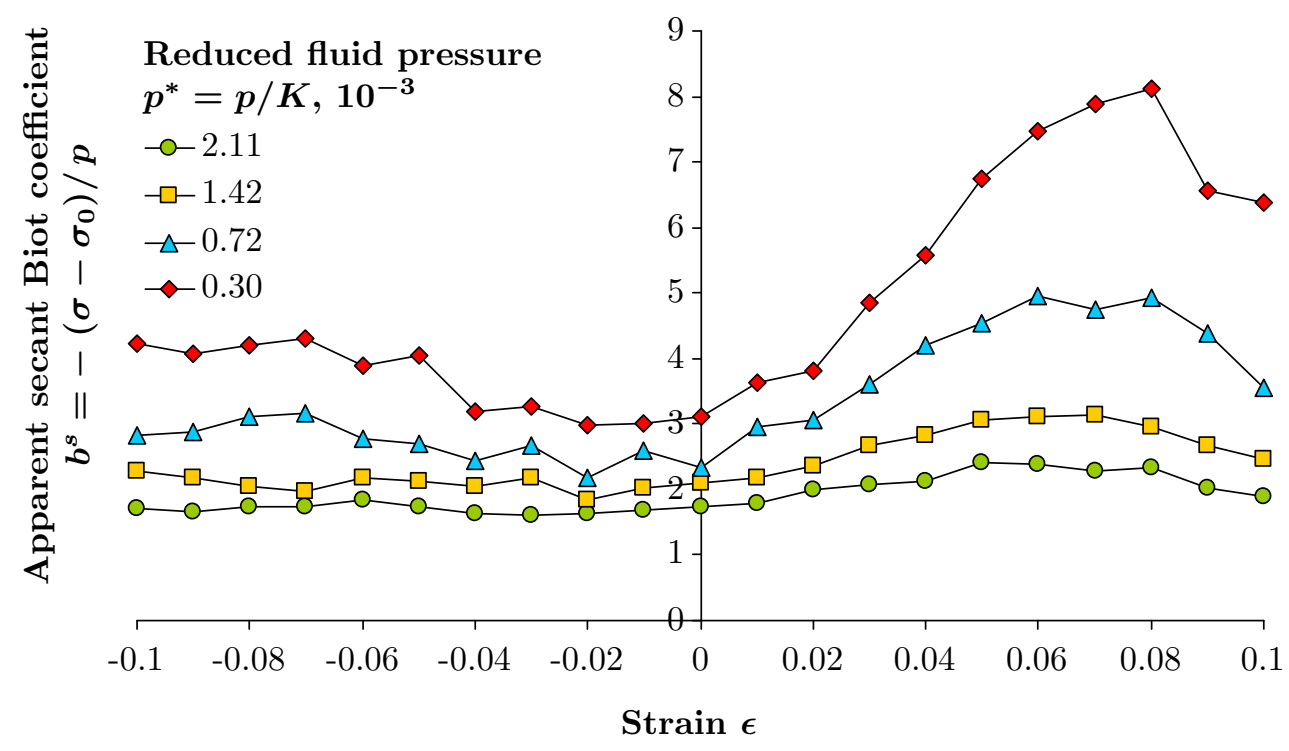

Figure 10: Apparent secant Biot coefficient $b^{s}$ of the amorphous chain.

and the number of fluid molecules per pore versus the size of the pores (considered as the distance between two atoms of the solid) for a fluid pressure $p^{*}=5.742 \times 10^{-3}$. We also sketch the filling of a slit pore in order to understand the different situations at stake.

- For pore sizes between 5.6 and $6.1 \AA$, the pore could accommodate 2 fluid molecules. The density remained almost constant and the fluid-induced stress mostly increased with the strain in this range, and reached a maximum at a pore size of about $6 \AA$. The fluid had a disjoining effect up to a pore size of $5.8 \AA$ and a joining effect in larger pores.

- For pore sizes between 6.1 and $6.6 \AA$, a third molecule may enter the pore. The density increased with the strain and the fluid-induced stress decreased with the strain. The fluid had a joining effect in pores smaller than $6.2 \AA$. For larger pores, the larger the pore, the more disjoining the fluid.

- Finally, for pore sizes between 5.4 and $5.6 \AA$, the second fluid molecule could be excluded from the pore. The density was an increasing function of the pore size and the fluid was strongly disjoining.

The mechanical effect of adsorption can be interpreted in terms of the commensurability of the size of the micropores to the size of the fluid molecules. The size of the pores for which the fluid-induced stress was maximal $(5.4 \AA)$ did not correspond to completely filled pores. Indeed, Eq. (16) shows that the fluid-induced stress depends on the derivative of the adsorbed amounts of fluid with respect to the strain (at the current and at lower chemical potentials). Therefore, the mechanical effect of adsorption in a crystalline microporous medium can not be simply related to the pore filling, but to the capacity of the pores to accommodate new molecules with strain.

The results displayed in Fig. 11 are consistent with simulations of disjoining pressure in slit pores available in the literature. Balbuena et al. (1993) computed the disjoining pressure of a Lennard-Jones fluid adsorbing in graphite slit pores. In the range of pores sizes we have in common, our results for a crystalline chain compare well with their results. Do et al. (2008) reported results of simulations of slit pores as well. They observed that fluid has a joining effect at small chemical potentials, but a disjoining one at high chemical potentials. 


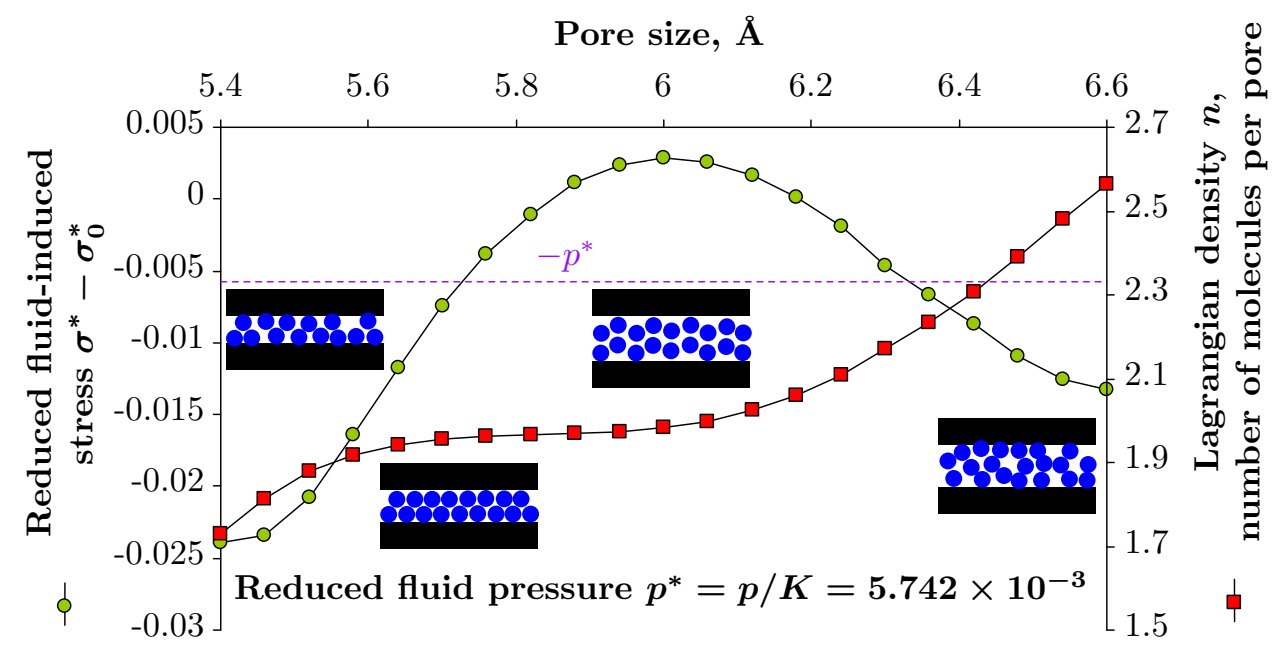

Figure 11: Fluid-induced stress and fluid density as a function of the pore size (considered as the average distance between two solid atoms) in the case of the crystalline chain.

The disjoining pressure isotherms obtained by Kowalczyk et al. (2008) (adsorption of argon between carbon walls) and Yang et al. (2010) (adsorption of methane in coal) exhibit only one main disjoining oscillation because of the high temperature they considered.

For the amorphous chain, we observed that the adsorbed amount increased linearly with the strain (see Fig. 8). Such a smooth behavior can be interpreted as a sum of all the complex responses of each specific pore size present in the amorphous chain. At a given bulk fluid pressure, some pores contract while others expand, which eventually leads, at the scale of the porous medium, to an overall disjoining action of the fluid, for any strain of the medium. Interestingly, even at low fluid pressure, we observed no joining effect of the fluid for the amorphous chain whereas, for the crystalline chain, a joining effect was observed for all strains considered. Such a discrepancy is due to the fact that the range of pore sizes explored in the crystalline chain (see Fig. 11) is too narrow to account for all pore sizes of the amorphous chain, which extend from $3 \AA$ up to $11 \AA$. Balbuena et al. (1993) showed that pores that are a few-Angstrom large (i.e., which are about the size of one fluid molecule) exhibit a very strong disjoining behavior upon adsorption, even at low fluid pressure. Moreover, for pores that are larger than 5 fluid molecules (i.e., larger than about $9 \AA$ in our case), the fluid-induced stress is negative.

An analysis of the data displayed in Fig. 8 shows that, for the amorphous chain, the adsorbed amount depended almost linearly on the strain of the medium, from what follows that the first-order expansion (37) of the adsorption isotherm should be valid for the amorphous chain in the range of strains here considered $(-0.1 \leq \epsilon \leq 0.1)$. In addition, based on the same data, a calculation of $\left(\partial n /\left.\partial \epsilon\right|_{p}\right) / n^{0}$ (where $n^{0}=n(\epsilon=0, p)$ ) shows that the coupling coefficient $C(p)$ introduced in Sec. 1.4 did almost not depend on the pressure $p$ of the fluid $(C(p)=1.52$ with a standard deviation of $12 \%$ on the whole range of pressures considered in this study), from what we conclude that the linearized constitutive equation (39) should hold for the amorpous chain. And, indeed, the fluid-induced stress calculated with the linearized constitutive equation (39) and displayed in Fig. 9 compares well with the fluid-induced stresses estimated directly from the molecular simulations with the Virial estimate. Such a good agreement proves that the linearized poroelastic constitutive equations derived in Sec. 1.4 enable to satisfactorily describe the poromechanical behavior of the amorpous chain.

From the results of the molecular simulations, for both the crystalline and the amorphous chains we computed the adsorbed amount $n^{u}(p)$ of fluid and the swelling $\epsilon^{u}(p)$ which one 


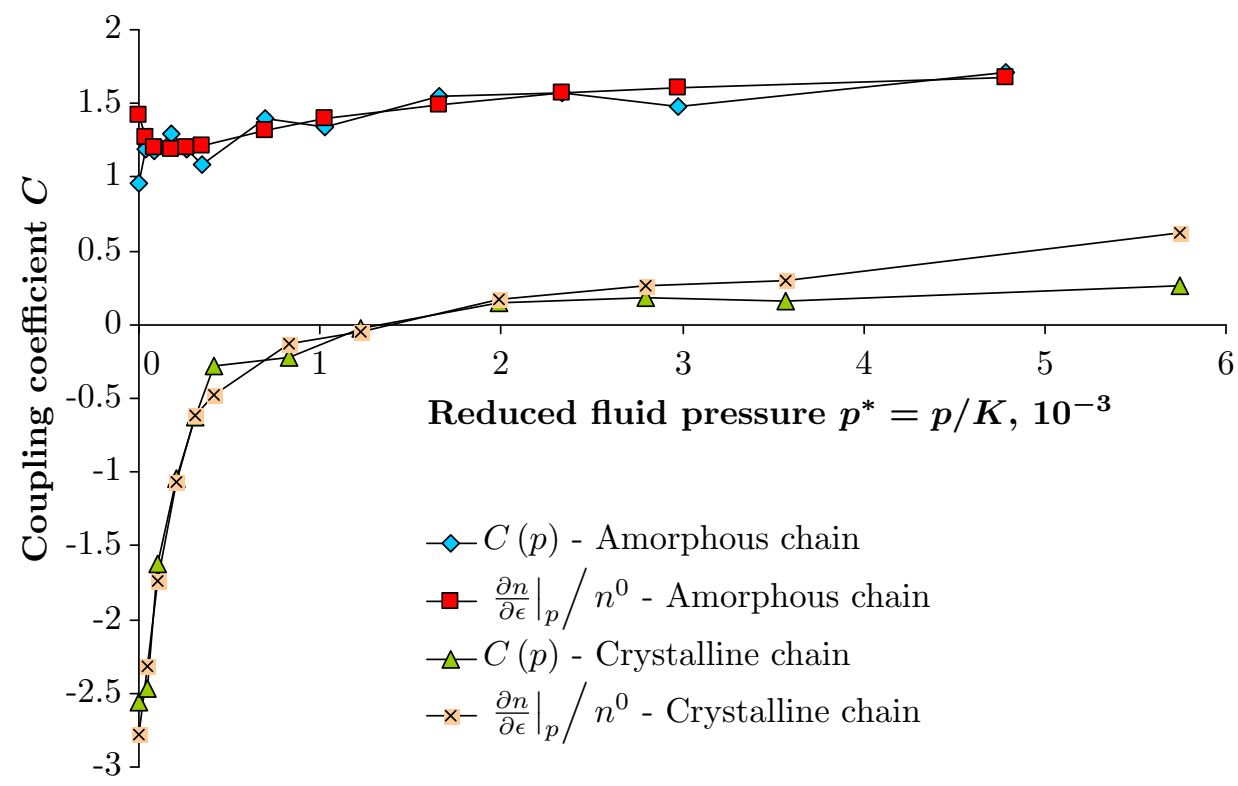

Figure 12: Coupling coefficient $C(p)$ estimated with Eq. (41) and as $\left(\partial n /\left.\partial \epsilon\right|_{p}\right) / n^{0}$.

would measure if he were to perform a regular adsorption experiment in which he would immerse the microporous medium in the fluid (in such a case, $\sigma=-p$ ). Then, making use of Eq. (41), we computed the coupling coefficient $C(p)$, which we display in Fig. 12 together with the coefficient $\left(\partial n /\left.\partial \epsilon\right|_{p}\right) / n(\epsilon=0, p)$. For both chains, the coupling coefficient $C(p)$ could equivalently be calculated with Eq. (41) or from its definition (37). As expected, the amorphous chain exhibited a nearly constant coupling coefficient $C(p)$. By contrast, for the crystalline chain the coupling coefficient $C(p)$ depended on the bulk fluid pressure, from what follows that the linearized poroelastic constitutive equation (39) does not apply for such a chain.

\section{Application to adsorption of methane in coal}

Coal is a disordered microporous medium with a variety of pore sizes whose molecular structure is amorphous (Jain et al., 2006a). In this section, we aim at verifying whether the poromechanical behavior of coal with methane can be satisfactorily described with the constitutive equations derived in Sec. 1.1. By analogy with the amorphous chain considered in Sec. 2.2, one can even wonder whether this poromechanical behavior can not be well described with the linearized constitutive equation (38) or (39). In order to answer those questions, this section is devoted to molecular simulations of adsorption of methane in a microporous carbon structure (called CS1000) representative of a realistic coal. In order to quantify the coupling between adsorption and strain numerically, the implemented carbon structure is compliant.

\subsection{Implementation of the molecular simulations}

Using the parameters described below, a first series of molecular simulations was performed to identify the volume of the unstressed carbon sample in the absence of any fluid. Then we applied various strains to the sample and let it relax for each strain with a Monte Carlo canonical simulation. The final molecular configuration obtained at each strain was used as a starting configuration for the simulations of adsorption of methane. The simulations 
of adsorption were performed in the canonical ensemble regarding the atoms of the CS1000 sample and in the grand canonical ensemble regarding the molecules of methane.

The coal microporous matrix was the CS1000 model (Jain et al., 2006b). This model is obtained by hybrid reverse Monte Carlo reconstruction methodology from a carbonated saccharose. CS1000 is made of atoms of carbon and hydrogen and exhibits an amorphous structure representative of the disordered microporosity found in coal. CS1000 is denser than real coal and does not include atoms of oxygen, whereas real coal does.

The carbon-carbon interactions within CS1000 were modeled with a modified Morse potential proposed by Belytschko et al. (2002) for hybridized $\mathrm{sp}^{2}$ atoms of carbon: such a potential should be well adapted to the study of coal, since most atoms of carbon in coal are hybridized $\mathrm{sp}^{2}$. Moreover, this potential is expected to provide realistic results for what concerns mechanical issues. The modified Morse potential is the sum of a conventional Morse potential and of a bond angle bending potential. In this study we only considered the second order term in the bond angle bending potential. The equilibrium distance in the Morse potential was equal to the average equilibrium distance between atoms of carbon in the CS1000 sample:

$$
U_{\text {Morse }}=D\left[\left(1-\exp \left(-a\left(r-r_{0}\right)\right)\right)^{2}-1\right] \text { and } U_{\text {angle }}=\frac{1}{2} k_{\theta}\left(\theta-\theta_{0}\right)
$$

where $r$ is the distance between two atoms of carbon, $r_{0}=1.423 \AA$ is the equilibrium distance between atoms of carbon, $D=6.03 \times 10^{-19} \mathrm{~J}$ and $a=2.625 \AA^{-1}$ are the parameters of the Morse potential, $\theta$ is the angle between three adjacent atoms of carbon, $\theta_{0}=2.094 \mathrm{rad}$ is the equilibrium angle, and $k_{\theta}=9.0 \times 10^{-19} \mathrm{~J}$ is the parameter for the bond angle bending potential.

Practically, the atoms of hydrogen within the solid skeleton have very little impact on the mechanical behavior of coal: this behavior is indeed mostly governed by the atoms of carbons and their interactions. Therefore, the potentials of interaction of atoms of hydrogen with atoms of carbon within the CS1000 sample had very little effect on the overall mechanical behavior of the sample. We chose those potentials in order to ensure the stability of the molecular structure. The carbon-hydrogen interaction within the CS1000 sample was modeled with a harmonic potential $U_{h}=k\left(r-r_{0}\right)^{2} / 2$, where $r_{0}=1.09 \AA$ is the equilibrium distance between atoms of carbon and of hydrogen, and where $k=8 \times 10^{-18} \mathrm{~J} . \AA^{-1}$ was such that the second-order derivative of this harmonic potential at equilibrium was the same as for the Morse potential for carbon-carbon interactions. We also accounted for the carboncarbon-hydrogen and hydrogen-carbon-hydrogen bond angle bending energies by using the same potential as for the carbon-carbon-carbon bond angles. Finally, we also modeled short range repulsive interactions between non-bonded atoms of hydrogen and of carbon by using the potential $U_{r}=4 \varepsilon(\sigma / r)^{12}$, where $\varepsilon=\sqrt{\varepsilon_{\mathrm{C}} \cdot \varepsilon_{\mathrm{H}}}$ and $\sigma=\left(\sigma_{\mathrm{C}}+\sigma_{\mathrm{H}}\right) / 2$ were obtained following the Lorentz-Berthelot rules with $\sigma_{\mathrm{C}}=3.36 \AA, \varepsilon_{\mathrm{C}}=28 \cdot k_{B}, \sigma_{\mathrm{H}}=2.42 \AA$, and $\varepsilon_{\mathrm{H}}=15.08 \cdot k_{B}$.

Two atoms of carbon of the CS1000 sample were considered bonded if the initial distance between them was less than $1.8 \AA$. This value is greater than the location of the first peak of the carbon-carbon pair correlation function of the initial CS1000 sample. Likewise, an atom of carbon and an atom of hydrogen were considered bonded if the initial distance between them was less than 1.4 A. During the simulation, no bond could be added or removed. From the initial CS1000 sample obtained by reconstruction, we removed 8 atoms of hydrogen which were bonded to no atom of carbon but to another atom of hydrogen: indeed, the reactive potential used by Brenner (1990) in order to obtain the initial CS1000 by hybrid reverse Monte Carlo reconstruction allowed for the creation of hydrogen-hydrogen bonds.

We checked that the CS1000 sample was stable with the potentials of interaction given above. In order to do so, we performed canonical simulations of CS1000 in the absence of any fluid and checked that atoms that were initially bonded would still verify the bond criteria 


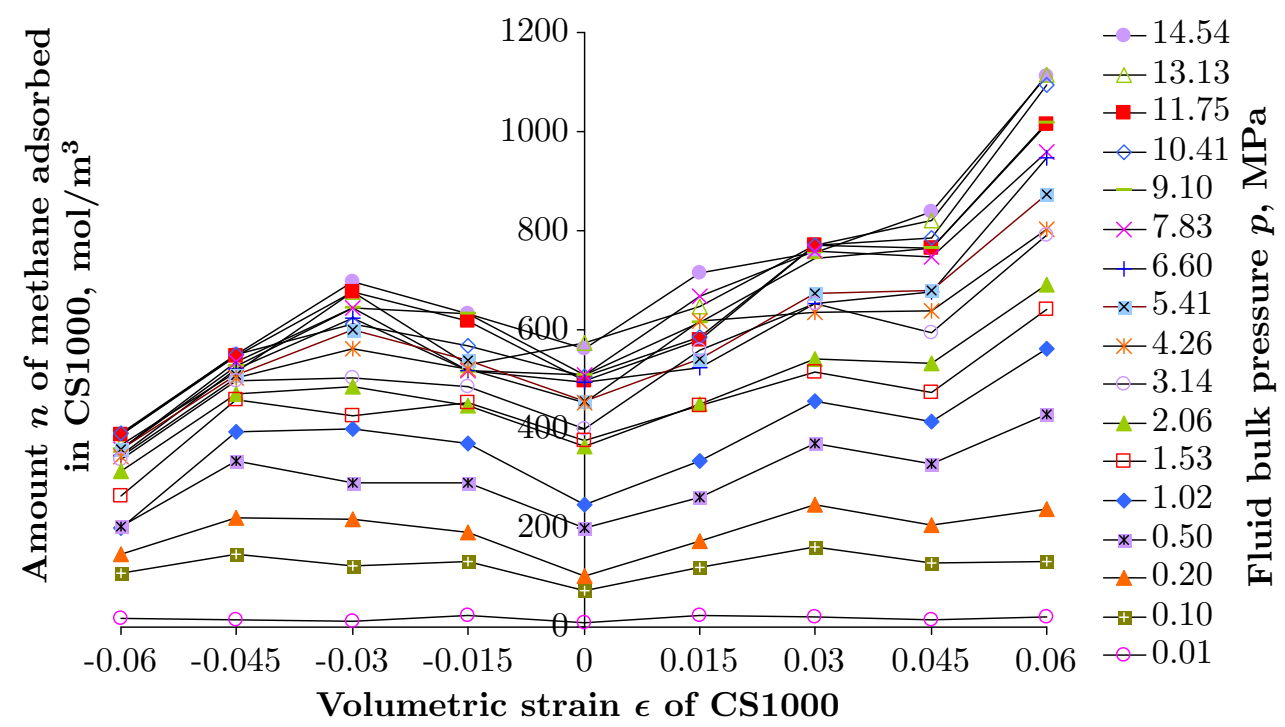

Figure 13: Adsorbed amount of methane in CS1000.

at the end of a simulation; that there was no non-bonded pair of atoms that would verify the bond criteria at the end of a simulation; and that the pair correlation functions before and after a simulation were similar.

During the simulations of adsorption of methane on CS1000, methane-methane interactions were governed by a Lennard-Jones potential with the parameters $\sigma_{\mathrm{CH}_{4}}=3.751 \AA$ and $\varepsilon_{\mathrm{CH}_{4}}=148 \cdot k_{B}$, which are suitable for methane in supercritical conditions (Kurniawan et al., 2006). The potentials of interaction between a molecule of methane and the atoms of the CS1000 sample were Lennard-Jones potentials as well. The parameters for methanecarbon and methane-hydrogen interactions were obtained following the Lorentz-Berthelot rules: $\sigma_{\mathrm{CH}_{4}-\mathrm{i}}=\left(\sigma_{\mathrm{CH}_{4}}+\sigma_{\mathrm{i}}\right) / 2$ and $\varepsilon_{\mathrm{CH}_{4}-\mathrm{i}}=\sqrt{\varepsilon_{\mathrm{CH}_{4}} \cdot \sigma_{\mathrm{i}}}$ with $\sigma_{\mathrm{C}}=3.36 \AA, \varepsilon_{\mathrm{C}}=28 \cdot k_{B}$, $\sigma_{\mathrm{H}}=2.42 \AA$ and $\varepsilon_{\mathrm{H}}=15.08 \cdot k_{B}$.

\subsection{Results and discussion}

We display in Fig. 13 the adsorbed amount of methane (per unit volume of undeformed porous medium) in function of the strain of the CS1000 sample for various methane bulk pressures. When the volumetric strain of the CS1000 sample was negative, the adsorbed amount of methane depended on the strain of the medium non-monotonically, which reminds of the response of the crystalline chain to adsorption (see Fig. 2). In contrast, when the volumetric strain of the CS1000 was positive, the adsorbed amount of methane increased mostly linearly with the strain of the medium, which reminds of the response of the amorphous chain to adsorption (see Fig. 8). Such an observation suggests that, at least for cases in which coal swells, the linearized poroelastic model derived in Sec. 1.4 should be applicable. Indeed, a calculation of $\left(\partial n /\left.\partial \epsilon\right|_{p}\right) / n^{0}$ (where $n^{0}=n(\epsilon=0, p)$ and where $\partial n /\left.\partial \epsilon\right|_{p}$ was averaged over all positive strains considered here) shows that the coupling coefficient $C(p)$ introduced in Sec. 1.4 did almost not depend on the pressure $p$ of the fluid $(C(p)=6.30$ with a standard deviation of $15 \%$ on the whole range of pressures considered in this study), thus proving that the linearized poroelastic constitutive equation is valid for coal, at least when coal swells.

The applicability of the linearized poroelastic constitutive equations to adsorption of methane in coal could also be checked directly with experimental data. In order to do so, we used the data of Ottiger et al. (2008), who performed $\mathrm{CH}_{4}$ adsorption experiments. 


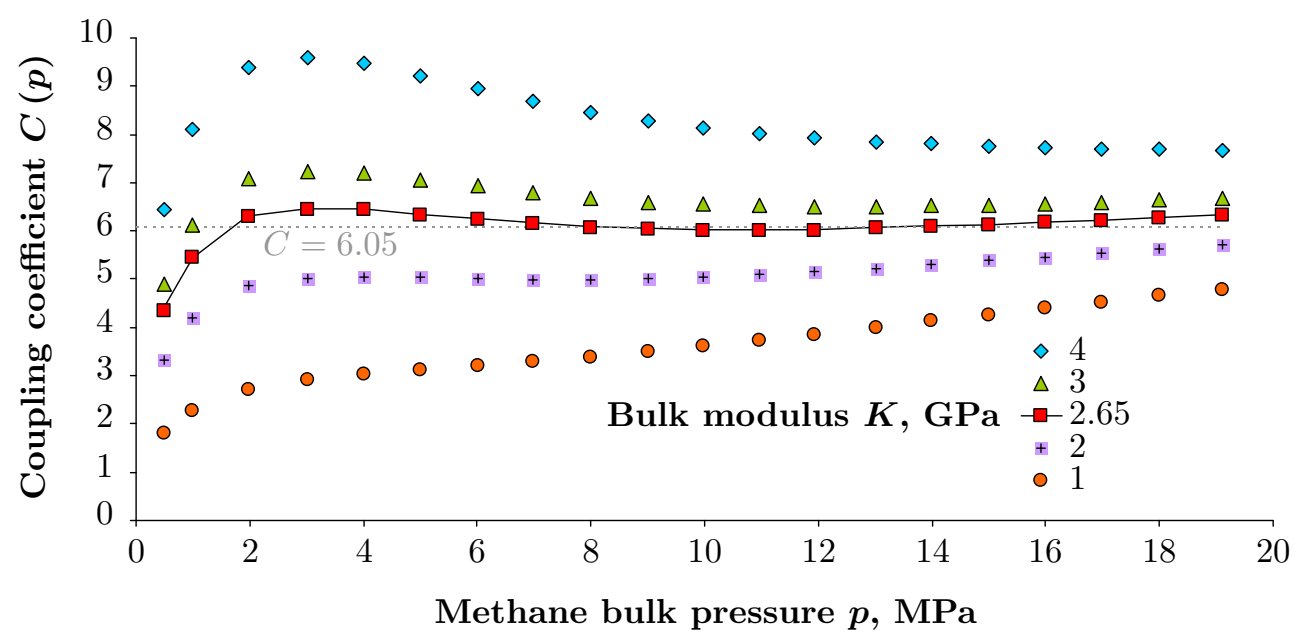

Figure 14: Coupling coefficient $C(p)$ estimated from the adsorption experiments of Ottiger et al. $(2006,2008)$

Over their adsorption experiments, they measured the swelling of the sample and the excess adsorbed amount (Ottiger et al., 2008), which we translated into a total adsorbed amount thanks to an estimate of the volume of micropores in the coal sample (Ottiger et al., 2006). In order to calculate the coupling coefficient $C(p)$ with Eq. (41), one needs to know the density of the sample and its bulk modulus $K$ in the absence of any fluid. We estimated the density of the coal sample to $1300 \mathrm{~kg} / \mathrm{m}^{3}$, which is a characteristic value for an average coal (Senel et al., 2001). We display in Fig. 14 the coupling coefficient $C(p)$ calculated for bulk moduli $K$ ranging from $1 \mathrm{GPa}$ to $4 \mathrm{GPa}$, which are plausible values for coal (Wang et al., 2009). The back-calculated coupling coefficient was the least pressure-dependent for a bulk modulus $K=2.65 \mathrm{GPa}$. For such a bulk modulus, we back-calculated a coupling coefficient $C(p)=6.05 \pm 7 \%$, i.e., a coupling coefficient $C(p)$ which practically did not depend on the bulk fluid pressure. This calculation suggests that the linearized poroelastic constitutive equation (39) can be applied to an adsorption of methane in coal.

We estimated the swelling that one would measure in a regular adsorption experiment if the poromechanical behavior of coal was exactly governed by the linearized poroelastic constitutive equation (39). The volumetric strain $\epsilon^{u}$ of a coal sample immersed in the fluid (in such a case, $\sigma=-p$ ) can be calculated from Eq. (39):

$$
\epsilon^{u}=-\frac{p}{K}+\frac{C}{K} \int_{0}^{p} n^{0}(p) \bar{V}_{b}(p) \mathrm{d} p
$$

We calculated this swelling by using $C=6.05, K=2.65 \mathrm{GPa}$, and by estimating the adsorbed amount $n^{0}(p)$ at zero strain from grand canonical Monte Carlo simulations of methane in a rigid CS1000 sample at $T=318.15 \mathrm{~K}$ (Vandamme et al., 2010). We display in Fig. 15 the results of this calculation together with the swelling measured experimentally by Ottiger et al. (2008) at $T=318.15 \mathrm{~K}$. Despite a small shift, which is due to the fact that the coupling coefficient $C(p)$ is not really constant at low fluid bulk pressures (see Fig. 14), both curves compare reasonably well, thus proving that the linearized constitutive equation (39) describes well the poromechanical behavior of coal subject to an adsorption of methane.

The coupling coefficient $C=6.05$ back-calculated from the adsorption experiments of Ottiger et al. (2008) compares well with the coupling coefficient $C=6.30$ back-calculated from the molecular simulations. Such a good agreement shows the relevance of the molecular simulations we performed. Nevertheless, the non-linear dependence of the adsorbed amount of methane with respect to the strain, which we observed in our numerical simulations when 


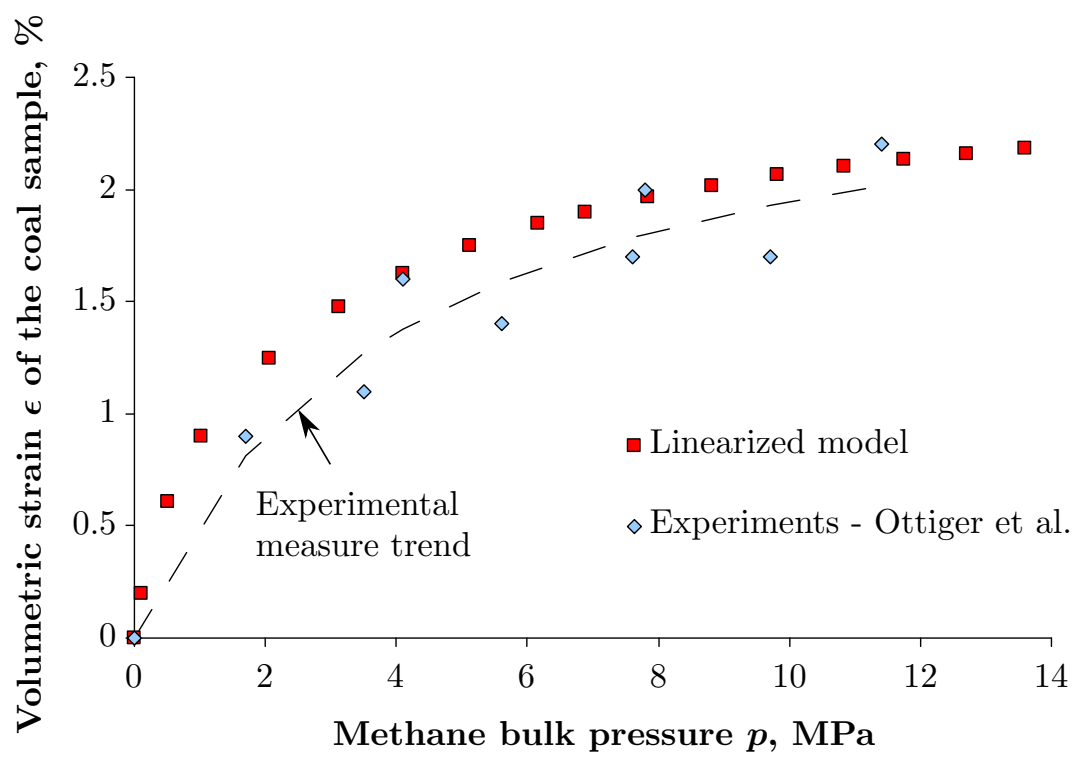

Figure 15: Volumetric strain of a coal sample immersed in methane. Comparison of the strain predicted by the linearized model with the strain measured experimentally (Ottiger et al., 2008).

the strain of the CS1000 sample was negative (see Fig. 13), is surprising. It is unclear whether such a dependence is a true property of the sample or an artifact of the simulations. Indeed, it may well be that the CS1000 sample was not representative enough to capture accurately the behavior of coal under compression: this molecular model is quite small $\left(25 \times 25 \times 25 \AA^{3}\right)$ and can accommodate only a few pores, which may not be sufficiently representative of the variety of pore sizes and shapes in real coal. On the other hand, it may well be that such a dependence is a true property of coal under compression: compression might narrow the pore size distribution, which would make the response of coal to adsorption evolve from a type exemplified by the amorphous chain (linear dependence of the adsorbed amount of fluid on the strain of the medium) toward a type exemplified by the crystalline chain (complex dependence of the adsorbed amount of fluid on the strain of the medium).

\section{Conclusions}

In this work, we derived poroelastic constitutive equations (Eqs. (16)-(18)) which are valid for a generic porous medium. Those equations are thus also valid for a porous medium with pores of nanometer size, in which all fluid molecules are in an adsorbed state. For such a microporous medium, in which the fluid molecules are not in their bulk state, usual poromechanics breaks down. We validated those constitutive equations by performing molecular simulations of adsorption of fluid in one-dimensional microporous media. Those simulations also showed that microporous media can exhibit a counterintuitive behavior: their drained bulk modulus can become strain-dependent because of adsorption, even when the medium behaves linearly in the absence of any fluid, and their Biot coefficient can take values greater than unity and smaller than zero.

The full determination of the poromechanical behavior of a porous medium requires the knowledge of how the amount of fluid in the porous medium depends on both the bulk pressure of the fluid and on the strain of the medium. But, often, the dependence of the isotherm of adsorption with respect to strain is disregarded since, for a macroporous medium, the amount of fluid in the system simply increases linearly with the strain. But, in a microporous medium, we showed that the adsorbed amount can depend in a complex manner on 
the strain and that this dependence can play a tremendous role on how the medium will respond mechanically to adsorption. At the scale of the micropore, we explained this nonlinear dependence by the commensurability of the size of the pore to the size of the fluid molecules.

Interestingly, we showed that, when the pore size distribution of the microporous medium is wide, the amount of fluid in the system increases linearly with the strain of the medium, although this response at the scale of the system results from a complex combination of closure and opening of micropores. In the case of a porous medium for which the amount of fluid would depend linearly on the strain of the medium, we derived a linearized constitutive equation (Eq. (38) or Eq. (39)).

Measuring isotherms of adsorption at different levels of strain of the sample would be very difficult. Practically, an adsorption experiment often consists in immersing the porous medium in a fluid and in measuring the amount of fluid adsorbed and, sometimes, the swelling of the sample. In such an experiment, the confining stress is equal to the opposite of the fluid pressure. Therefore, a regular adsorption experiment provides only partial information on how the isotherms of adsorption depend on the strain of the medium. In the general case, this information is not sufficient to fully characterize the poromechanical behavior of the porous medium. But, if this poromechanical behavior can be well described by the linearized constitutive equation we derived, then the regular adsorption experiment provides enough information. We described how to check, from a regular adsorption experiment, whether the linearized constitutive equation (38) or (39) can apply to the porous medium and to the fluid considered.

As an application, we considered the case of an adsorption of methane in coal. We showed, both by molecular simulations and from experimental data of a regular adsorption experiment, that the response of coal to adsorption can be well described with the linearized constitutive equation (39) we derived. Such a possibility is a consequence of the disordered structure of coal, which is a microporous material with a large variety of pore sizes. Therefore, our work provides the constitutive equations that are relevant to the study of the poromechanics of coal. Those constitutive equations are relevant for any other porous media, and in particular for microporous media in which adsorption induces a significant mechanical response.

\section{Acknowledgments}

M. Vandamme thanks Prof. A.V. Neimark for the interesting discussions they had.

\section{Bibliography}

Allen, M.P., Tildesley, D.J., 1989. Computer Simulation of Liquids. Oxford University Press, Inc., New York, NY.

Bae, J.S., Bhatia, S.K., 2006. High-pressure adsorption of methane and carbon dioxide on coal. Energy \& Fuels 20 (6), 2599-2607.

Balbuena, P.B., Berry, D., Gubbins, K.E., 1993. Solvation pressures for simple fluids in micropores. The Journal of Physical Chemistry, 97 (4), 937-943.

Belytschko, T., Xiao, S.P., Schatz, G.C., Ruoff, R.S., 2008. Atomistic simulations of nanotube fracture. Phys. Rev. B, 65 (23), 235430.

Biot, M., 1941. General theory of three-dimensional consolidation. J. Appl. Phys., 12 (2), 155-164.

Brenner, D.W., 1990. Empirical potential for hydrocarbons for use in simulating the chemical vapor deposition of diamond films. Phys. Rev. B, 42 (15), 9458-9471. 
Coudert, F.-X., Jeffroy, M., Fuchs, A. H., Boutin, A., Mellot-Draznieks, C., 2008. Thermodynamics of Guest-Induced Structural Transitions in Hybrid Organic-Inorganic Frameworks. Journal of the American Chemical Society, 130 (43), 14294-14302.

Coussy, O., 1995. Mechanics of Porous Continua. Wiley, Hoboken, NJ.

Coussy, O., 2004. Poromechanics. Wiley, Hoboken, NJ.

Coussy, O., 2010. Mechanics and Physics of Porous Solids. Wiley, Hoboken, NJ.

Do, D.D., Nicholson, D., Do, H.D., 2008. Effects of Adsorbent Deformation on the Adsorption of Gases in Slitlike Graphitic Pores: A Computer Simulation Study. The Journal of Physical Chemistry C, 112 (36), 14075-14089.

Edmiston, P.L., Underwood, L.A., 2009. Absorption of dissolved organic species from water using organically modified silica that swells. Separation and Purification Technology $66(3), 532-540$.

Frenkel, D., Smit, B., 2001. Understanding Molecular Simulation. Academic Press, Inc., San Diego, CA.

Gibbs, J.W., 1928. The Collected Works of J. Willard Gibbs. Longmans, Green and Co, New York, NY.

Gor, G.Y., Neimark, A.V., 2011. Adsorption-Induced Deformation of Mesoporous Solids: Macroscopic Approach and Density Functional Theory. Langmuir 27 (11), 6926-6931.

Grosman, A., Ortega, C., 2008. Influence of elastic deformation of porous materials in adsorption-desorption process: A thermodynamic approach. Physical Review B 78 (8).

Grosman, A., Ortega, C., 2009. Influence of Elastic Strains on the Adsorption Process in Porous Materials: An Experimental Approach. Langmuir 25 (14), 8083-8093.

Harpalani, S., Schraufnagel, A., 1990. Measurement of parameters impacting methane recovery from coal seams. Geotechnical and Geological Engineering 8 (4), 369-384.

Jain, S.K., Gubbins, K.E., Pellenq, R.J.M., Pikunic, J.P., 2006. Molecular modeling and adsorption properties of porous carbons. Carbon 44 (12), 2445-2451.

Jain, S.K., Pellenq, R.J.M., Pikunic, J.P., Gubbins, K.E., 2006. Molecular modeling of porous carbons using the hybrid reverse Monte Carlo method. Langmuir 22 (24), 9942-9948.

Kowalczyk, P., Ciach, A., Neimark, A.V., 2008. Adsorption-induced deformation of microporous carbons: Pore size distribution effect. Langmuir 24 (13), 6603-6608.

Kurniawan, Y., Bhatia, S.K., Rudolph, V., 2006. Simulation of binary mixture adsorption of methane and CO 2 at supercritical conditions in carbons. AIChE Journal 52 (3), 957967.

Levine, J.R., 1996. Model study of the influence of matrix shrinkage on absolute permeability of coal bed reservoirs. Geological Society, London, Special Publications 109 (1), 197-212.

Malani, A., Ayappa, K.G., Murad, S., 2009. Influence of Hydrophilic Surface Specificity on the Structural Properties of Confined Water. The Journal of Physical Chemistry B, 113 (42), 13825-13839.

Mazumder, S., Wolf, K.H., 2008. Differential swelling and permeability change of coal in response to CO2 injection for ECBM. International Journal of Coal Geology 74 (2), 123-138.

Murray, L.J., Dinca, M., Long, J.R., 2009. Hydrogen storage in metal-organic frameworks. Chemical Society Reviews 38 (5), 1294-1314.

Mushrif, S.H., Rey, A.D., 2009. An integrated model for adsorption-induced strain in microporous solids. Chemical Engineering Science 64, 4744-4753.

Neimark, A.V., Coudert, F.X., Triguero, C., Boutin, A., Fuchs, A.H., Beurroies, I., Denoyel, R., 2011. Structural Transitions in MIL-53 (Cr): View from Outside and Inside. Langmuir 27 (8), 4734-4741.

Ottiger, S., Pini, R., Storti, G., Mazzotti, M., 2008. Competitive adsorption equilibria of $\mathrm{CO} 2$ and $\mathrm{CH} 4$ on a dry coal. Adsorption, 14 (4), 539-556.

Ottiger, S., Pini, R., Storti, G., Mazzotti, M., Bencini, R., Quattrocchi, F., Sardu, G., Deriu, G., 2006. Adsorption of pure carbon dioxide and methane on dry coal from the sulcis coal province (SW Sardinia, Italy). Environ. Prog., 25 (4), 355-364. 
Pellenq, R.J.-M., Levitz, P.E., 2002. Capillary condensation in a disordered mesoporous medium: a grand canonical Monte Carlo study. Molecular Physics 100 (13), 2059-2077.

Pijaudier-Cabot, G., Vermorel, R., Miqueu, C., Mendiboure, B., 2011. Revisiting poromechanics in the context of microporous materials. Comptes Rendus Mécanique 339 (12), 770778.

Ravikovitch, P.I., Neimark, A.V., 2006. Density Functional Theory Model of Adsorption on Amorphous and Microporous Silica Materials. Langmuir 22 (26), 11171-11179.

Rice, J.R., Cleary, M.P., 1976. Some basic stress diffusion solutions for fluid-saturated elastic porous-media with compressible constituents. Reviews of Geophysics 14 (2), 227-241.

Senel, A.G., Guruz, A.G., Yucel, H., Kandas, A.W., Sarofim, A.F., 2001. haracterization of Pore Structure of Turkish Coals. Energy \& Fuels, 15 (2), 331-338.

Shuttleworth, R., 1950. The surface tension of solids. Proceedings of the Physical Society. Section A 63, 444-457.

Vandamme, M., Brochard, L., Lecampion, B., Coussy, O., 2010. Adsorption and strain: The CO2-induced swelling of coal. Journal of the Mechanics and Physics of Solids, 58 (10), 1489-1505.

Wang, G.X., Massarotto, P., Rudolph, V., 2009. An improved permeability model of coal for coalbed methane recovery and $\mathrm{CO} 2$ geosequestration. International Journal of Coal Geology, 77 (1-2), 127-136.

Yang, K., Lu, X., Lin, Y., Neimark, A. V., 2010. Deformation of Coal Induced by Methane Adsorption at Geological Conditions. Energy \& Fuels, 24 (11), 5955-5964. 